\title{
Biological Systems \\ as Reactive Systems
}

\section{Luca Cardelli}

Microsoft Research

Cambridge UK

2006-01-26 LMU Munich

www. luca.demon.co.uk 


\section{Years of Molecular Cell Biology}

- Genes are made of DNA

- Store digital information as sequences of 4 different nucleotides

- Direct protein assembly through RNA and the Genetic Code

- Proteins (>10000) are made of amino acids

- Process signals

- Activate genes

- Move materials

- Catalyze reactions to produce substances

- Control energy production and consumption

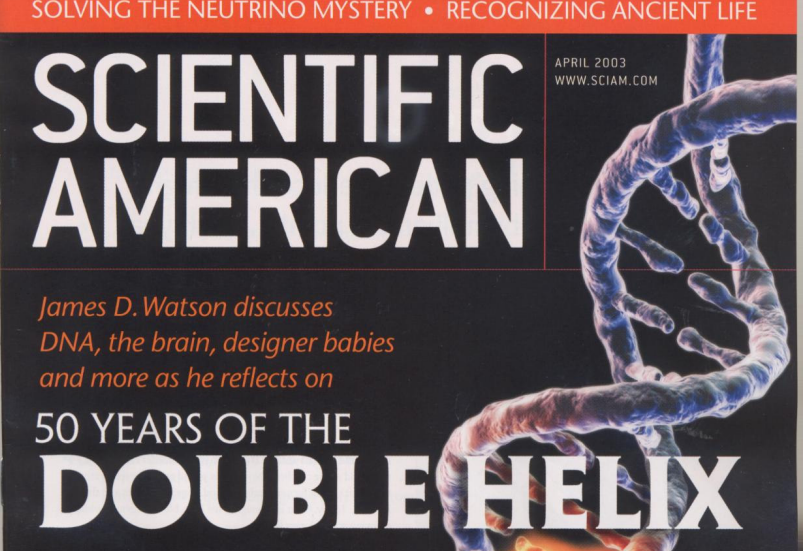

- Bootstrapping still a mystery

- DNA, RNA, proteins, membranes are today interdependent. Not clear who came first

- Separation of tasks happened a long time ago

- Not understood, not essential 


\section{Towards Systems Biology}

- Biologists now understand many of the cellular components

- A whole team of biologists will typically study a single protein for years

- Reductionism: understand the components in order to understand the system

- But this has not led to understand how "the system" works

- Behavior comes from complex patterns of interactions between components

- Predictive biology and pharmacology still rare

- Synthetic biology still unreliable

- New approach: try to understand "the system"

- Experimentally: massive data gathering and data mining (e.g. Genome projects)

- Conceptually: modeling and analyzing networks (i.e. interactions) of components

- What kind of a system?

- Just beyond the basic chemistry of energy and materials processing...

- Built right out of digital information (DNA)

- Based on information processing for both survival and evolution

- Highly concurrent

- Can we fix it when it breaks?

- Really becomes: How is information structured and processed? 


\section{Storing Processes}

- Today we represent, store, search, and analyze:

- Gene sequence data

- Protein structure data

- Metabolic network data

\section{Cellular Abstractions: Cells as Computation}

Regev\&Shapiro NATURE vol 419, 2002-09-26, 343

- Signaling pathway data

$-\ldots$

- How can we represent, store, and analyze biological processes?

- Scalable, precise, dynamic, highly structured, maintainable representations for systems biology.

- Not just huge lists of chemical reactions or differential equations.

- In computing...

- There are well-established scalable representations of dynamic reactive processes.

- They look more or less like little, mathematically based, programming languages. 


\section{Structural Architecture}

\section{Eukaryotic} Cell

(10 100 trillion in human body)

\section{Membranes everywhere}

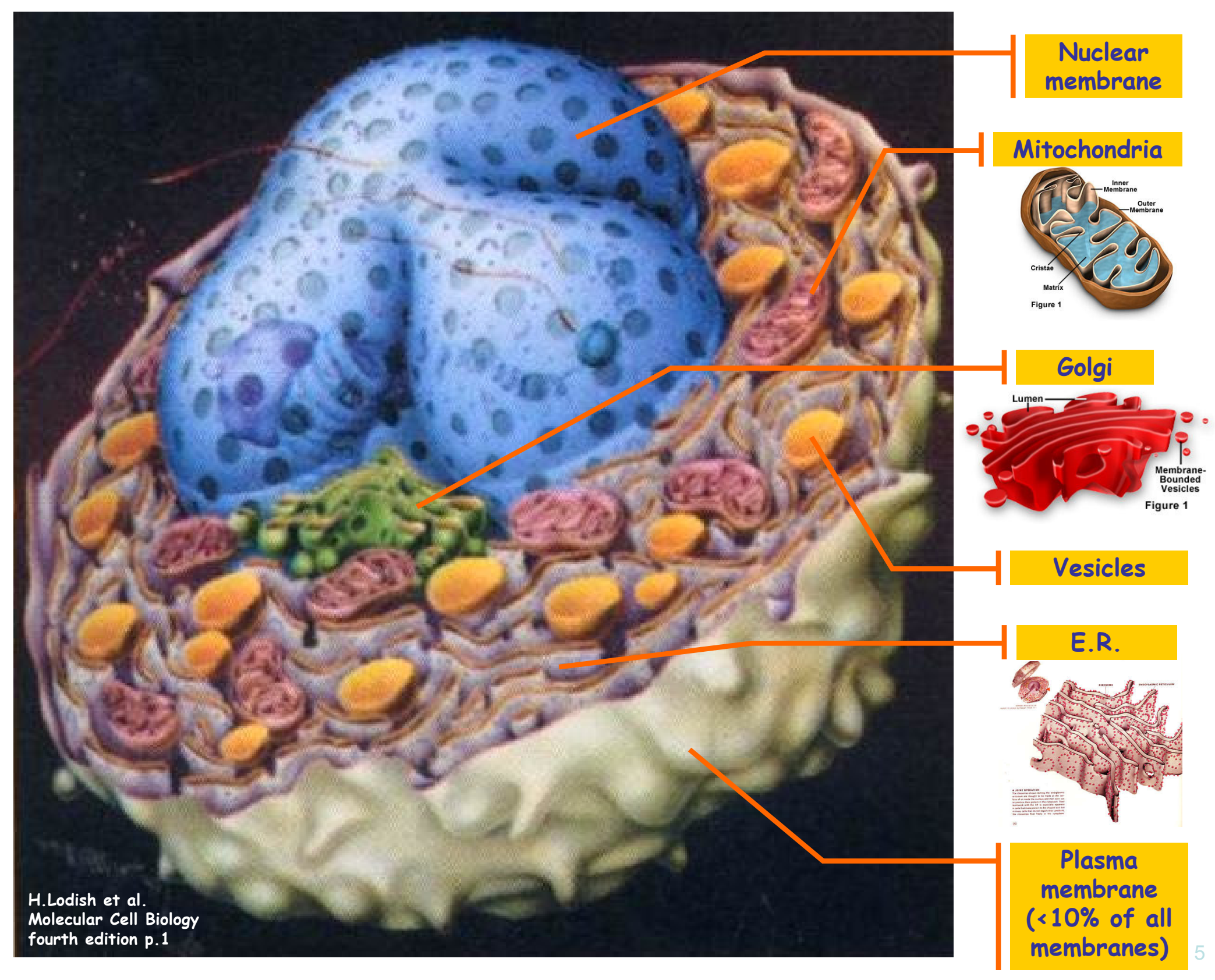




\section{Abstract Machines of Systems Biology}

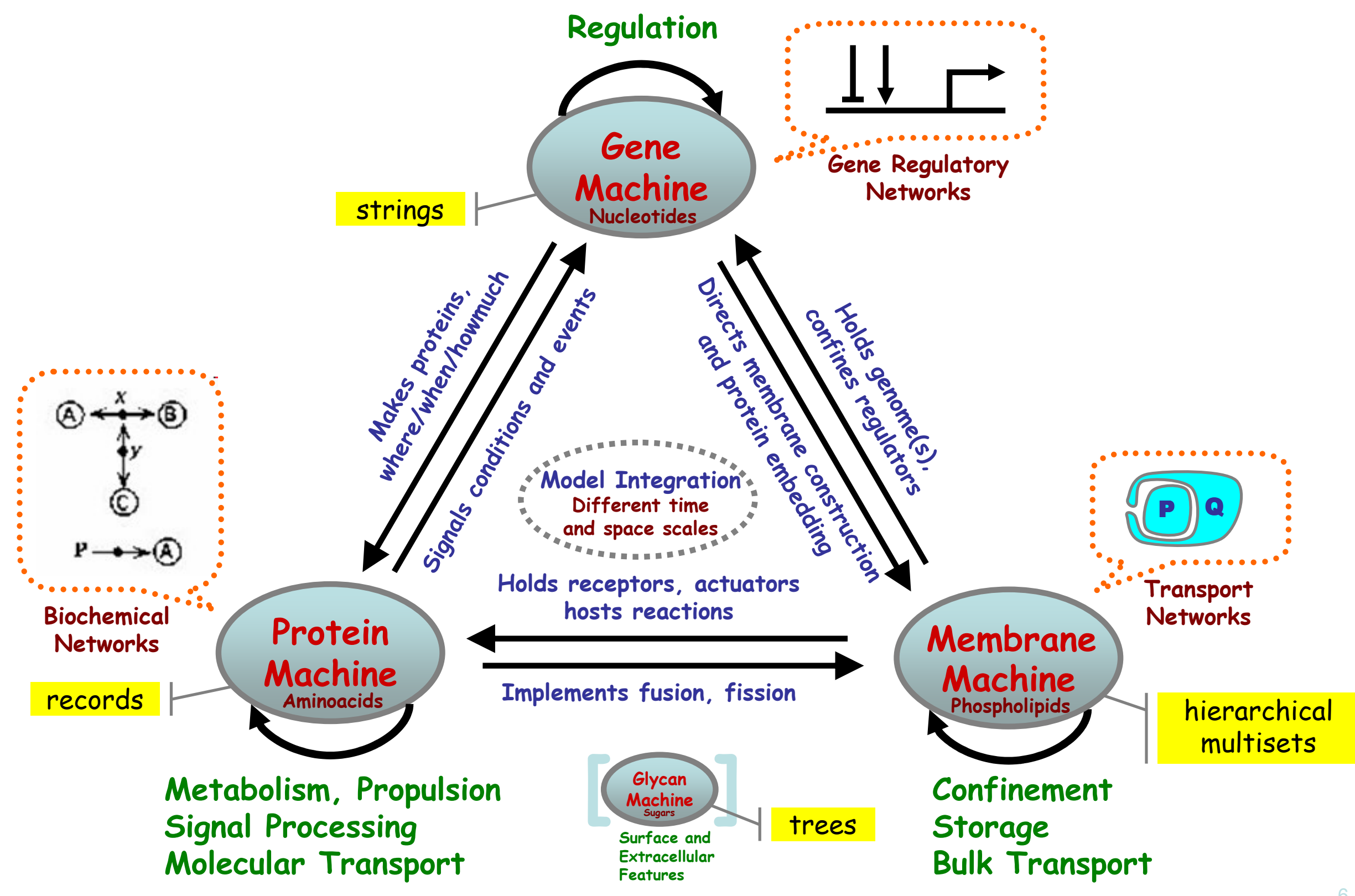




\section{Reactive Systems}

- Modeling biological systems

- Not as continuous systems (often highly nonlinear)

- But as discrete reactive systems; abstract machines where:

- States represent situations

- Event-driven transitions between states represent dynamics

- The adequacy of describing (discrete) complex systems as reactive systems has been argued convincingly [Harel]

- Many biological systems exhibit features of reactive systems:

- Discrete transitions between states

- Deep layering of abstractions ("steps" at multiple levels)

- Complexity from combinatorial interaction of simple components

- High degree of concurrency and nondeterminism

- "Emergent behavior" not obvious from part list 


\section{Chemistry vs. $\pi$-calculus}

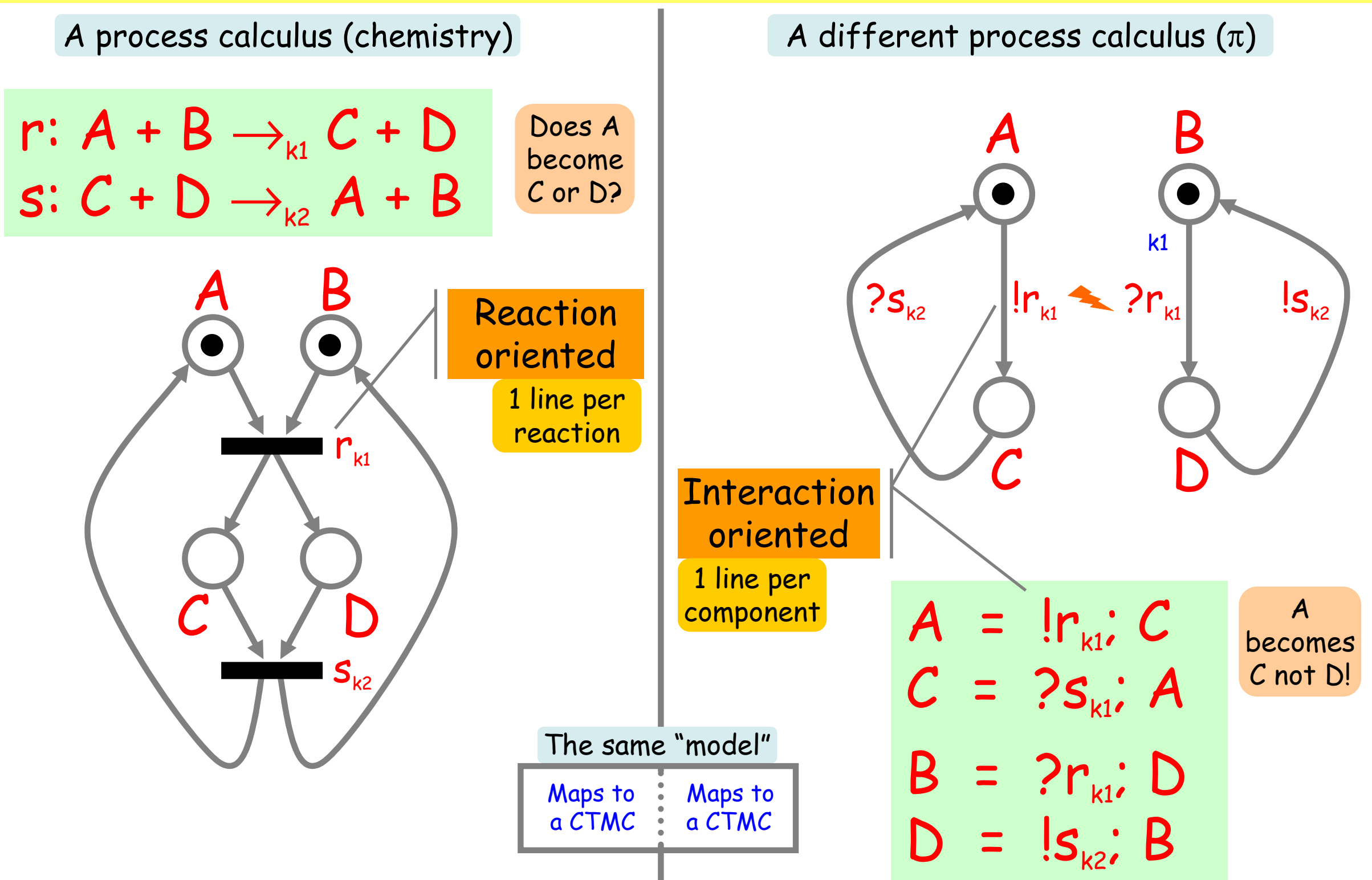

A Petri-Net-like representation. Precise and dynamic but not modular, scalable, or maintainable.

A compositional graphical representation (precise, dynamic and modular) and the corresponding calculus. 


\section{The Protein Machine Very close to the atoms.}

- Complex folded-up shapes that:

- Fit together, dock, undock.

- Excite/unexcite, warp each other.

- Bring together, catalyze, transform materials.

- Form complex aggregates and networks.

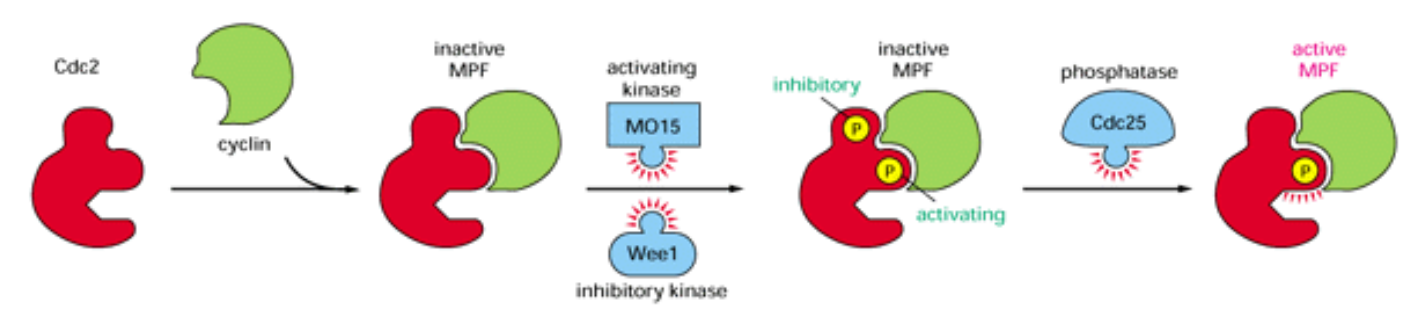

- Mapping out such networks:

- In principle, it's "just" a very large set of chemical equations.

- Notations have been developed to summarize and abstract.

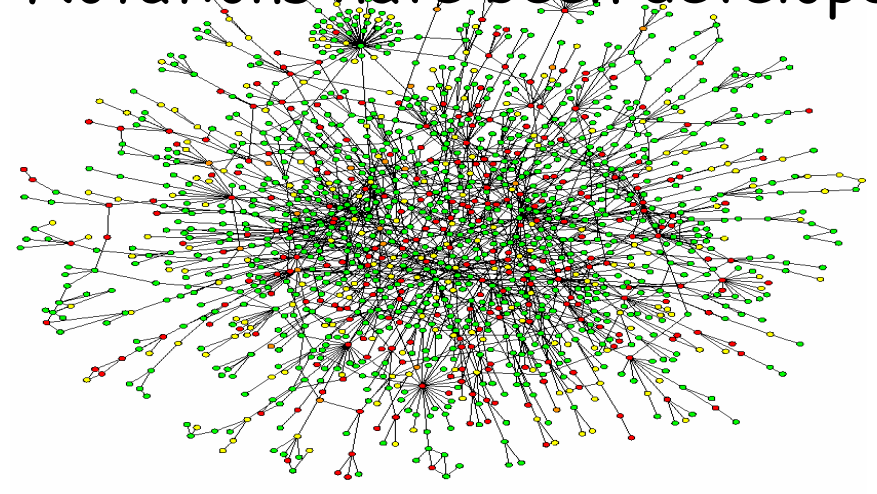




\section{Protein Structure}

Primary



The 20 Aminoacids

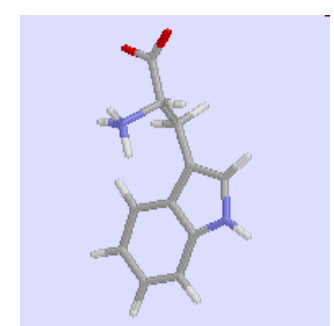

Tryptophan

\section{Secondary}
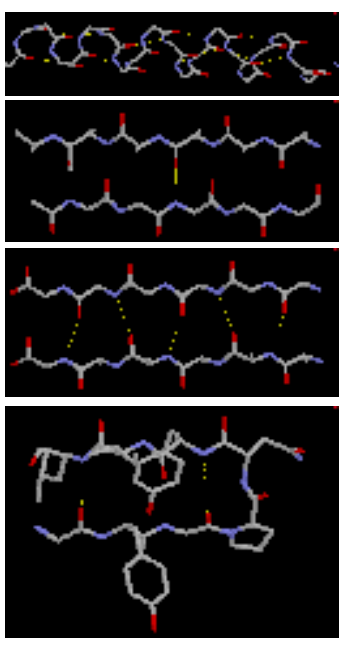

Alpha Helix, Beta Sheet

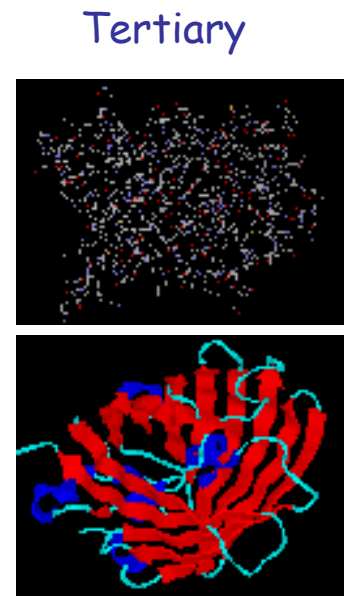

Green Fluorescent Protein



Triose Phosphate Isomerase 


\section{Protein Function}

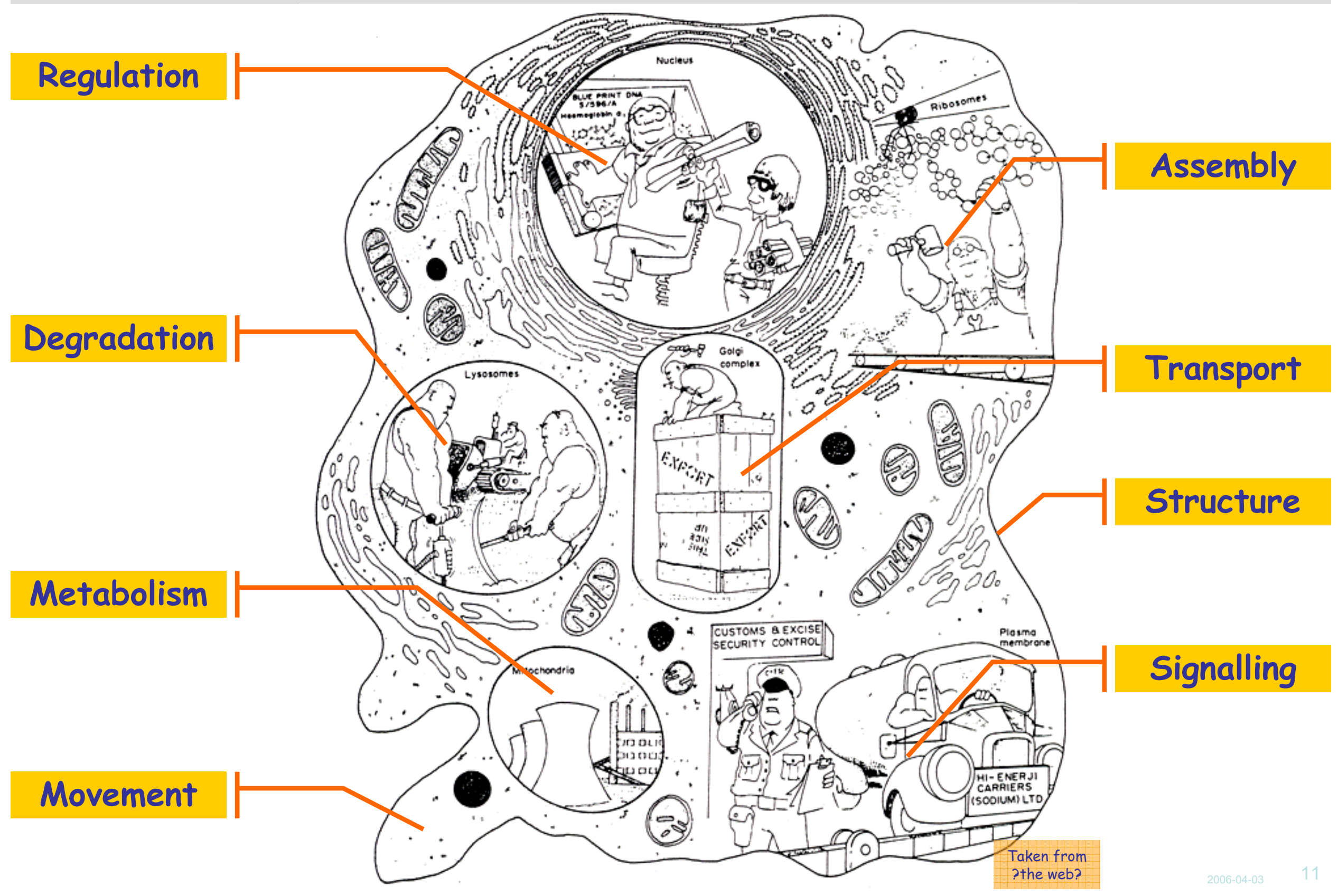




\section{MIM: Molecular Interaction Maps (Kohn)}

(A) $\leftrightarrow$ (B) Ttw double-arrowed line indicales ltat joudeins A and $\mathbf{B}$ call bind to each other. The "noude" placed ou the line represents the A:B complex.

(B) $\longleftrightarrow$ (B) Asymntetric binding where protein $A$ donates a peptide that binds to a receptor site or puckel on protejn $B$.

(A)
Oepresentation of umltimolecular complexes: $\lambda$ is A:B;
of components in a complex

Proteolytic cleavage at a specific site within a protein.

(A) $\longrightarrow$ B) Stoichiometric conversion of $\mathbf{A}$ into $\mathbf{B}$.

cylosol nuckeus Transport of A from cylosol to nucleus. The node

(A)

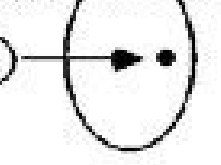
represents $\mathbf{A}$ after it has been transported into the nucleus.

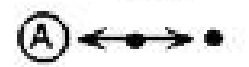

Formarion of a homodimer. Filled circle on the right represents another copy of $\mathbf{A}$. The node on the line represents the homodimer $\mathbf{A}: \mathbf{A}$.

$\stackrel{z \quad y}{\longrightarrow} z$ is the combination of states defined by $x$ and $y$.

- Enzymatic stimulation of a reaction.

$\longrightarrow$ General symbol for stimulation.

A bar behind the arrowhead signifies necessity.

1 General symbol for inhibition.

$\longrightarrow \quad$ Shorthand symbol for transcriptional activation.

Shorthand symbol for transcriptional inhibition.

Q Degradation products 


\section{Molecular Interaction Maps}

http://www.cds.caltech.edu/ hsauro/index.htm

JDesigner

The p53-Mdm2 and DNA Repair Regulatory Network

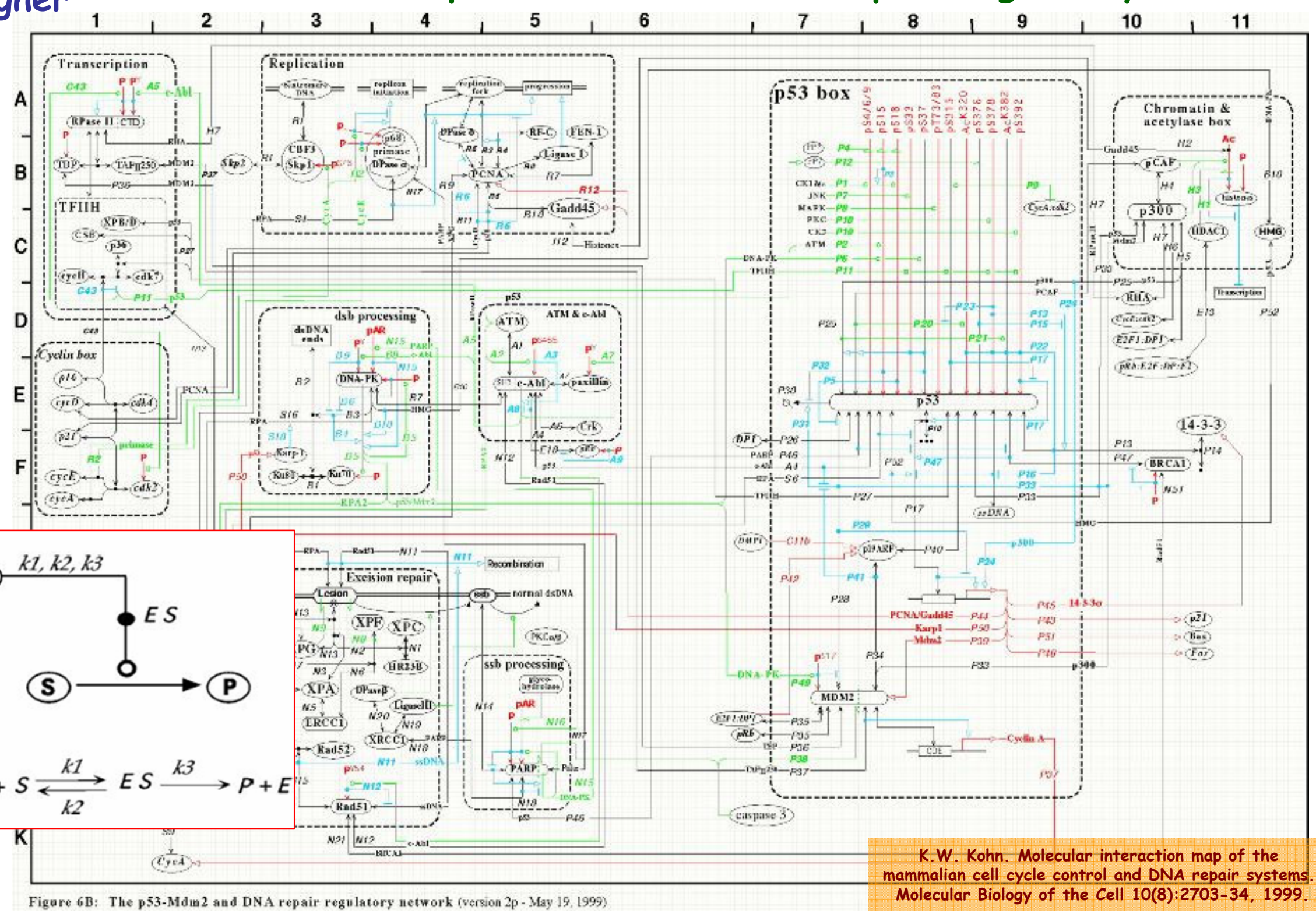




\section{The Protein Machine "Instruction Set"}

On/Off switches

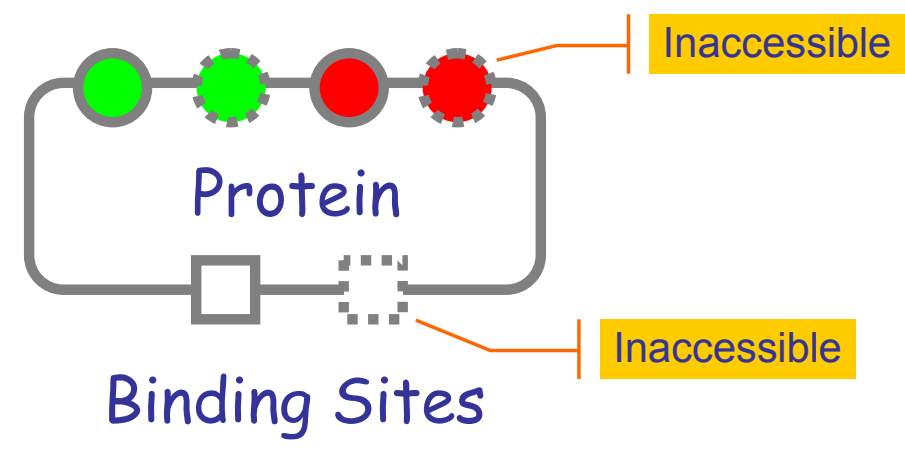

cf. BioCalculus [Kitano\&Nagasaki], $\kappa$-calculus [Danos\&Laneve] Each protein has a structure of binary switches and binding sites. But not all may be always accessible.

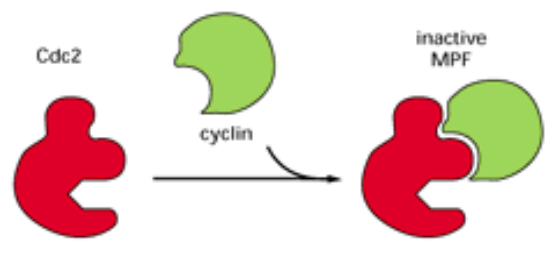


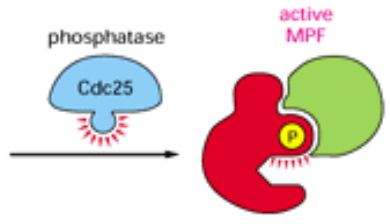
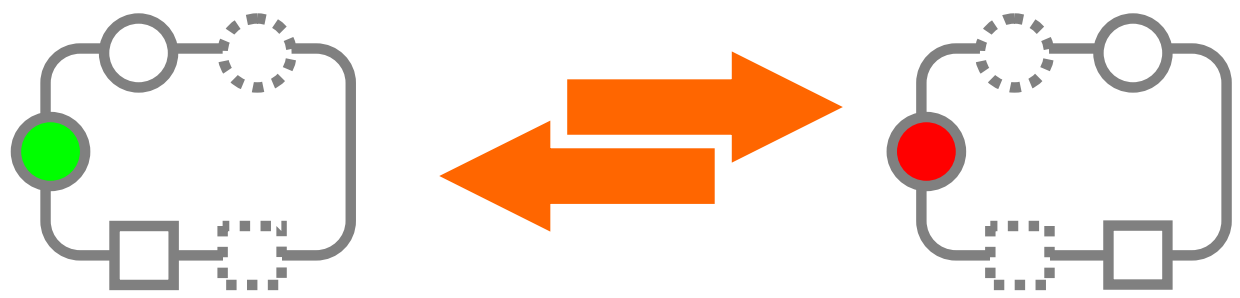

Switching of accessible switches.

- May cause other switches and

binding sites to become (in)accessible.

- May be triggered or inhibited by nearby specific proteins in specific states.
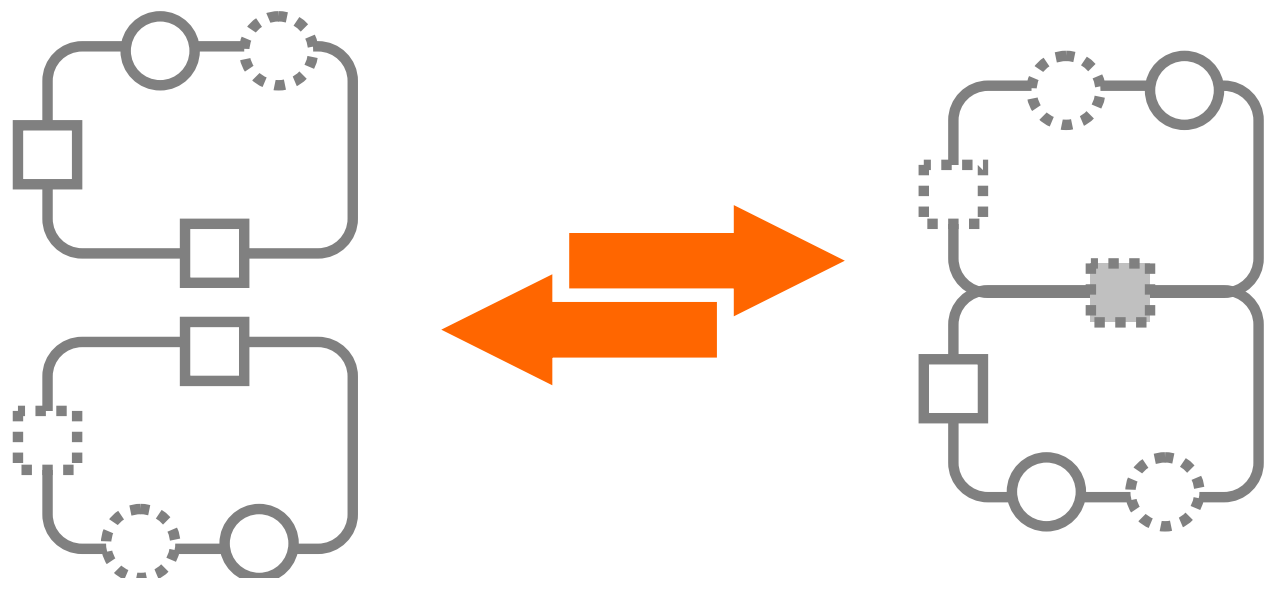

Binding on accessible sites.

- May cause other switches and

binding sites to become (in)accessible.

- May be triggered or inhibited by nearby specific proteins in specific states. 


\section{Notations for the Protein Machine}

- Stochastic $\pi$-Calculus

- Priami (following Hillston's PEPA) formalizes a stochastic version of $p$-calculus where channels have communication rates.

- BioSPi

- Regev-Shapiro-Silverman propose modeling chemical interactions (exchange of electrons and small molecules) as "communication".

- Standard stochastic simulation algorithms (Gillespie) can be used to run in-silico experiments.

- Complex formation is encoded via p-restriction.

- PEPA

- Calder Gilmore and Hillston model the ERK pathway.

- $k$-calculus

- Danos and Laneve (following Kitano's BioCalculus) define a calculus where complex formation is primitive.

- (Stochastic) Petri Nets

- S.Reddy'94 modeling pathways.

- Srivastava Perterson and Bentley analyze and simulate E.coli stress response circuit.
- Bio State Charts

- Harel uses State Charts to model biological interactions via a semi-graphical FSM notation.

- Pathway Logic

- Talcott-Eker-Knapp-Lincoln use term-rewriting.

- BioCham

- ChabrierRivier-Fages-Soliman use term-rewriting and $C L T$ modelchecking.

- Kohn Diagrams, Kitano Diagrams

- SBML (Systems Biology Markup Language)

- XML dialect for MIM's:

- Compartments (statically nested)

- Reagents with concentrations

- Reactions with various rate laws

- Read and written by many tools via the Systems Biology Workbench protocol 


\section{MAPK Cascade}

\section{Ultrasensitivity in the mitogen-activated protein cascade, Chi-Ying F. Huang}

and James E. Ferrell, Jr., 1996, Proc. Natl. Acad. Sci. USA, 93, 10078-10083.

Biochemistry: Huang and Ferrell

Proc. Natl. Acad. Sci. USA 93 (1996)

Table 2. Predicted Hill coefficients for MAP kinase cascade components: Varying the assumed $K_{\mathrm{m}}$ values

\begin{tabular}{|c|c|c|c|c|}
\hline \multirow[b]{2}{*}{ Reaction } & \multirow{2}{*}{$\begin{array}{c}\text { Range of assumed } K_{\mathrm{m}} \\
\text { values }\end{array}$} & \multicolumn{3}{|c|}{$\begin{array}{l}\text { Range of effective Hill-oetficients }(\mathrm{nH}) \\
\text { predicted for }\end{array}$} \\
\hline & & IMAPKKK & MAPKK & MAPK \\
\hline 1. MAPKKK $\rightarrow$ MAPKKK $*$ & $60-1500 \mathrm{nM}$ & 1.0 & 1.7 & 4.9 \\
\hline 2. MAPKKK $* \rightarrow$ MAPKKK & $601500 \mathrm{nM}$ & 1.0 & 1.7 & 4.9 \\
\hline 3. MAPKK $\rightarrow$ MAPKK-P & $60-1500 \mathrm{nM}$ & 1.0 & $1.3-2.3$ & $4.0-5.1$ \\
\hline 4. MAPKK-P $\rightarrow$ MAPKK & $60-1500 \mathrm{nM}$ & 1.0 & $1.5-1.9$ & $3.6-6.7$ \\
\hline 5. MAPKK-P $\rightarrow$ MAPKK-PP & $60-1500 \mathrm{nM}$ & 1.0 & $1.3-2.4$ & $3.8-5.2$ \\
\hline 6. MAPKK-PP $\rightarrow$ MAPKK-P & $60-1500 \mathrm{nM}$ & 1.0 & $1.7-1.8$ & $4.1-6.4$ \\
\hline 7. MAPK $\rightarrow$ MAPK-P & $60-1500 \mathrm{nM}\left(300 \mathrm{nM}^{\dagger}\right)$ & 1.0 & 1.7 & $3.7-6.2$ \\
\hline 8. MAPK-P $\rightarrow$ MAPK & $60-1500 \mathrm{nM}$ & 1.0 & 1.7 & $4.3-5.2$ \\
\hline 9. MAPK-P $\rightarrow$ MAPK-PP & $60-1500 \mathrm{nM}$ & 1.0 & 1.7 & $3.4-6.1$ \\
\hline 10. MAPK-PP $\rightarrow$ MAPK-P & $60-1500 \mathrm{nM}$ & 1.0 & 1.7 & $4.7-5.1$ \\
\hline
\end{tabular}

The assumed $K_{\mathrm{m}}$ values for each reaction were individually varied over the ranges shown, with the assumed $K_{\mathrm{m}}$ values for the other nine reactions held constant. The effective Hill coefficients were calculated from the steepness of the predicted stimulus/response curves, as described in the text.

The $K_{\mathrm{m}}$ value for reaction 7 has been measured to be $300 \mathrm{nM}$ for the phosphorylation of a mammalian MAPK by a MAPKK (N. Ahn, personal communication). All of the other $K_{\mathrm{m}}$ values were initially assumed to be $300 \mathrm{nM}$ as well

Calculations. Eqs. 1-10 represent the reactions of the MAPK cascade, which are shown schematically in Fig. 1 . We have use
Goldbeter and Koshland's nomenclature for the rate constantsthe letter a denotes association, $\mathrm{d}$ denotes dissociation withou catalysis, and $\mathrm{k}$ denotes product formation (11). KKK deno

$$
\begin{aligned}
& \mathrm{KKK}+\mathrm{E} 1 \underset{\mathrm{d}_{1}}{\stackrel{\mathrm{a}_{1}}{\rightleftarrows}} \mathrm{KKK} \cdot \mathrm{E} 1 \stackrel{\mathrm{k}_{1}}{\longrightarrow} \mathrm{KKK} *+\mathrm{E} 1 \\
& \mathrm{KKK}^{*}+\mathrm{E} 2 \underset{\mathrm{d}_{2}}{\stackrel{\mathrm{a}_{2}}{\rightleftarrows}} \mathrm{KKK} \cdot \mathrm{E} 2 \stackrel{\mathrm{k}_{2}}{\rightarrow} \mathrm{KKK}+\mathrm{E} 2 \\
& \mathrm{KK}+\mathrm{KKK}^{*} \underset{\mathrm{d}_{3}}{\stackrel{\mathrm{a}_{3}}{\rightleftarrows}} \mathrm{KK} \cdot \mathrm{KKK}^{*} \stackrel{\mathrm{K}_{3}}{\longrightarrow} \mathrm{KK}-\mathrm{P}+\mathrm{KKK}^{*} \\
& \text { KK-P + KK P' ase } \underset{d_{4}}{\stackrel{a_{4}}{\rightleftarrows}} \text { KK-P.KK P'ase } \\
& \stackrel{\mathrm{k}_{4}}{\rightarrow} \mathrm{KK}+\mathrm{KK} \mathrm{P}^{\prime} \text { ase }
\end{aligned}
$$



$$
\text { KK-PP + KK P'ase } \underset{d_{6}}{\stackrel{a_{6}}{\rightleftarrows}} \text { KK-PP·KK P'ase }
$$$$
\stackrel{\mathrm{k}_{6}}{\longrightarrow}
$$$$
\stackrel{\mathrm{k}_{6}}{\longrightarrow} \mathrm{KK}-\mathrm{P}+\mathrm{KK} \mathrm{P}^{\prime} \text { ase }
$$

$\mathrm{KK}-\mathrm{PP}+\mathrm{K} \underset{\mathrm{d}_{7}}{\stackrel{\mathrm{a}_{7}}{\rightleftarrows}} \mathrm{KK}-\mathrm{PP} \cdot \mathrm{K} \stackrel{\mathrm{K}_{7}}{\longrightarrow} \mathrm{KK}-\mathrm{PP}+\mathrm{K}-\mathrm{P}$

$\mathrm{K}-\mathrm{P}+\mathrm{K}$ P'ase $\underset{\mathrm{d}_{8}}{\stackrel{\mathrm{a}_{8}}{\rightleftarrows}} \mathrm{K}-\mathrm{P} \cdot \mathrm{K} \mathrm{P}^{\prime}$ ase $\stackrel{\mathrm{k}_{8}}{\longrightarrow} \mathrm{K}+\mathrm{K} \mathrm{P}^{\prime}$ ase

$\mathrm{K}-\mathrm{P}+\mathrm{KK}-\mathrm{PP} \underset{\mathrm{d}_{9}}{\stackrel{\mathrm{a}_{9}}{\rightleftarrows}} \mathrm{K}-\mathrm{P} \cdot \mathrm{KK}-\mathrm{PP} \stackrel{\mathrm{k}_{9}}{\longrightarrow} \mathrm{K}-\mathrm{PP}+\mathrm{KK}-\mathrm{PP} \quad$ [9]

K-PP + K P'ase $\underset{\mathrm{d}_{10}}{\stackrel{\mathrm{a}_{10}}{\rightleftarrows}}$ KK-PP·K P'ase

$\stackrel{\mathrm{k}_{10}}{\longrightarrow} \mathrm{K}-\mathrm{P}+\mathrm{K} \mathrm{P}^{\prime}$ ase
Reservoirs

Back Enzymes

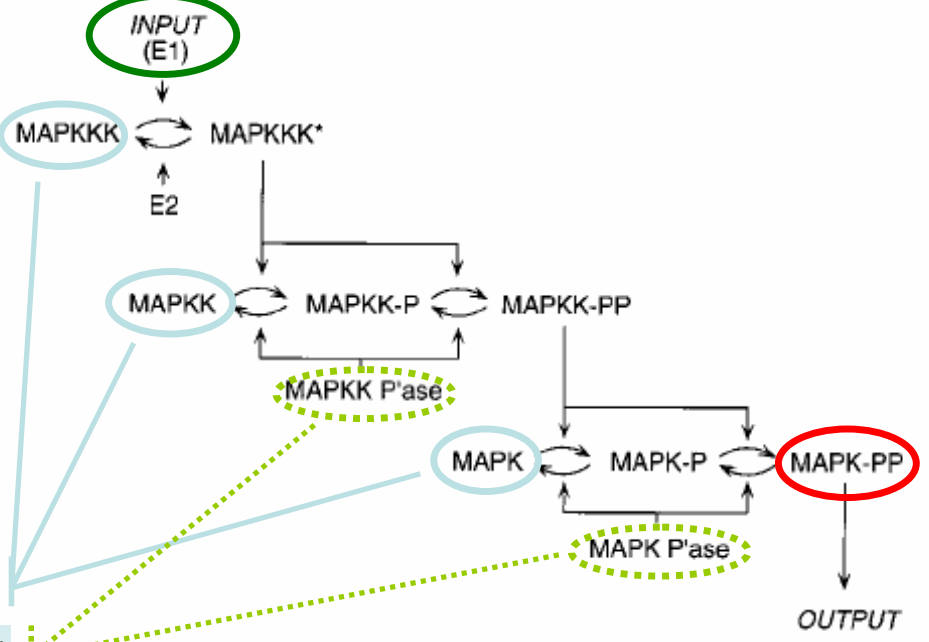

FIG. 1. Schematic view of the MAPK cascade. Activation of MAPK depends upon the phosphorylation of two conserved sites [Thr-183 and Tyr-185 in rat p42 MAPK/Erk2 $(4,5)]$. Full activation of MAPKK also requires phosphorylation of two sites [Ser-218 and Ser-222 in mouse Mek-1/MKK1 (6-10)]. Detailed mechanisms for the activation of various MAPKKKs (e.g., Raf-1, B-Raf, Mos) are not yet established; here we assume that MAPKKKs are activated and inactivated by enzymes we denote E1 and E2. MAPKKK* denotes activated MAPKKK. MAPKK-P and MAPKK-PP denote singly and doubly phosphorylated MAPKK, respectively. MAPK-P and MAPK-PP denote singly and doubly phosphorylated MAPK. P'ase denotes phosphatase.

\section{0 chemical reactions}




\section{The Circuit}

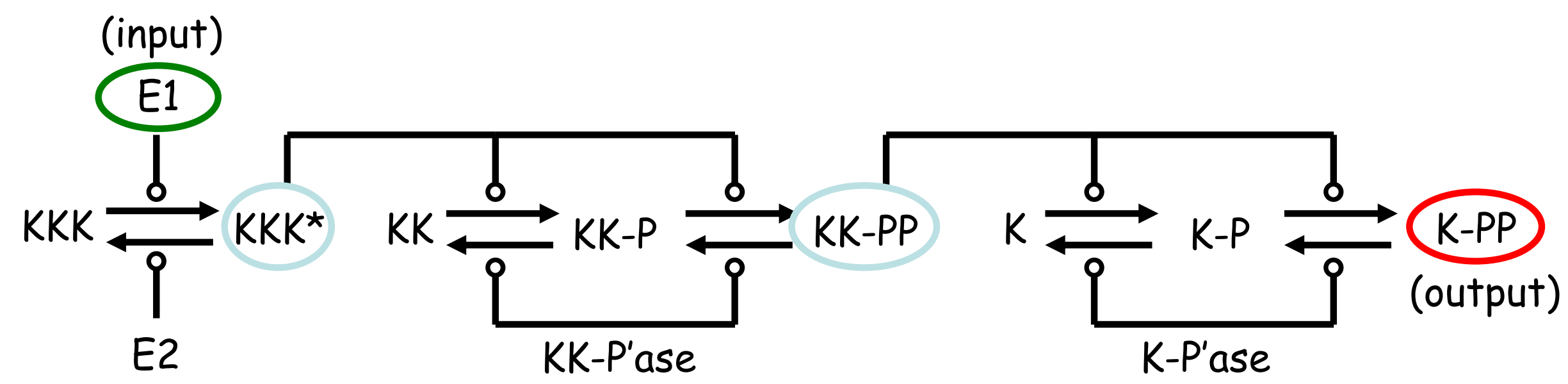




\section{Enzymatic Reactions}

Reaction View

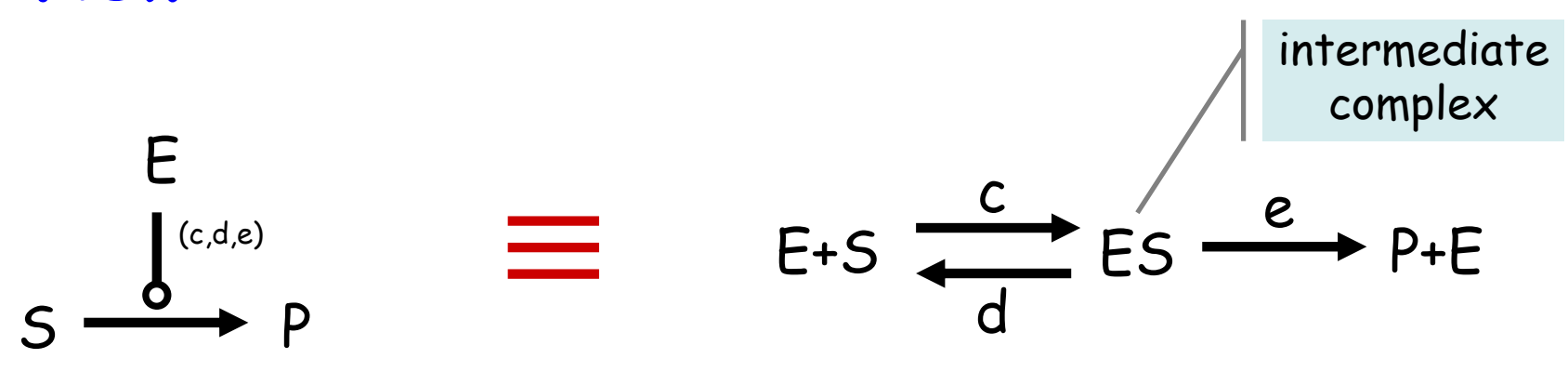

\section{Interaction View}

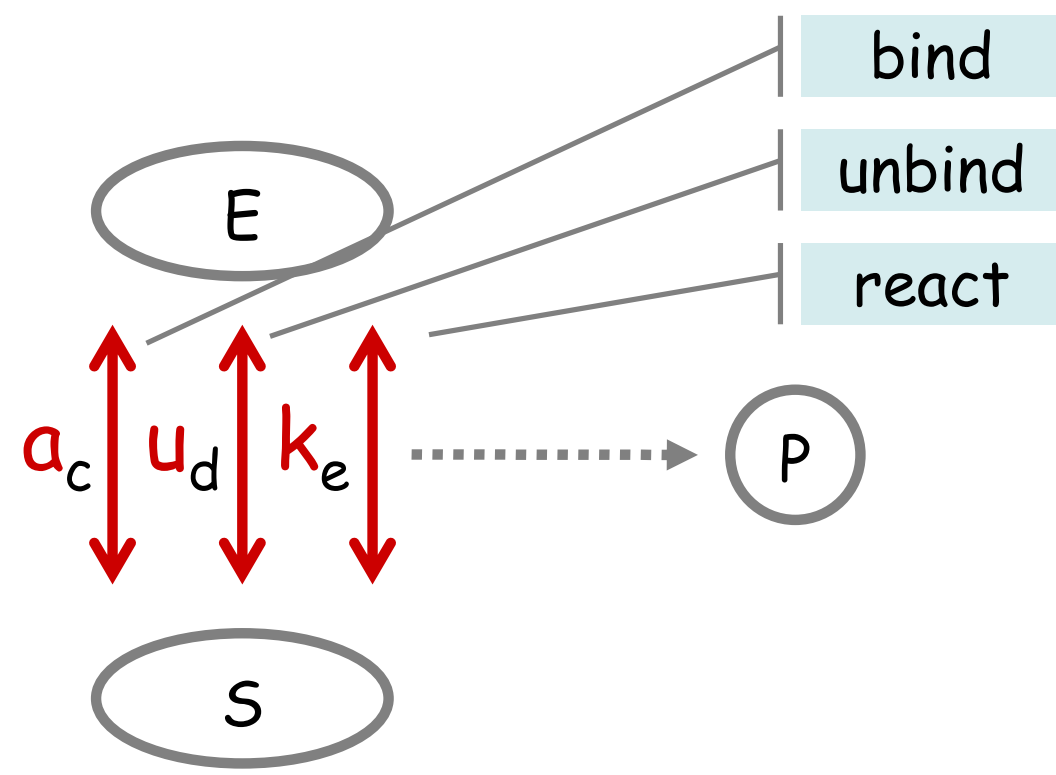

private bindings between one $S$ and one $E$ molecule
$S() \triangleq$ new u@d new k@e $! a_{c}(u, k) ;\left(! u_{d} ; S()+! k_{e^{\prime}} P()\right)$

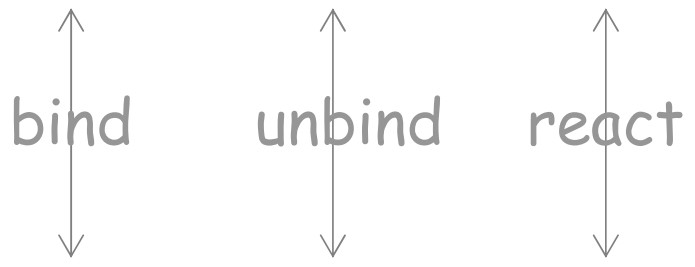

$E() \triangleq ? a_{c}(u, k) ;\left(? u_{d} ; E()+? k_{e^{\prime}} ; E()\right)$

$P() \triangleq \quad \cdots$ 


\section{MAPK Cascade Simulation in SPiM}
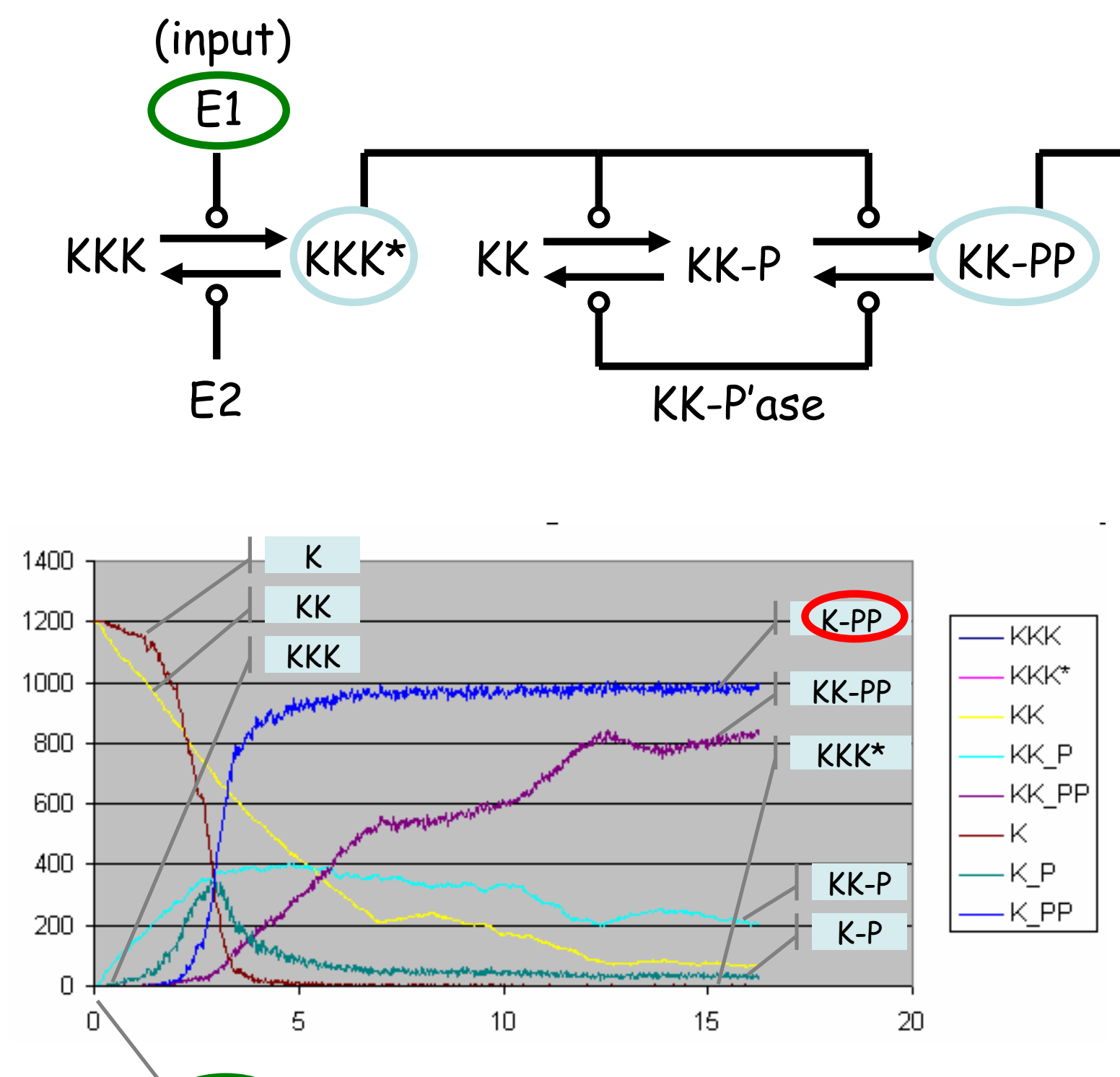

$1^{\text {st }}$ stage:

$\mathrm{KKK}^{\star}$ barely rises

$2^{\text {nd }}$ stage:

KK-PP rises, but is not stable

$3^{\text {rd }}$ stage:

K-PP flips up to max

even anticipating $2^{\text {nd }}$ stage

Rates and concentrations from paper:

1xE2 (0.3 nM)

$1 \times$ KKPase $(0.3 n M)$

$120 \times K P a s e(120 \mathrm{nM})$

$3 \times K K K(3 n M)$

$1200 \times K K(1.2$ uM)

$1200 \times K(1.2$ uM)

1xE1 injected

$d x=r x=150, a x=1$

$(K m x=(d x+r x) / a x, K m=300 n M)$ 


\section{MAPK Cascade Simulation in SPiM}


\section{All coefficients 1.0 !!!}

$100 \times K K K, 100 \times K K, 100 \times K$,

$13 \times E 2,13 \times K K P s e, 13 \times K P$ se.

$n \times E 1$ as indicated

(1xE1 is not sufficient to produce an output)
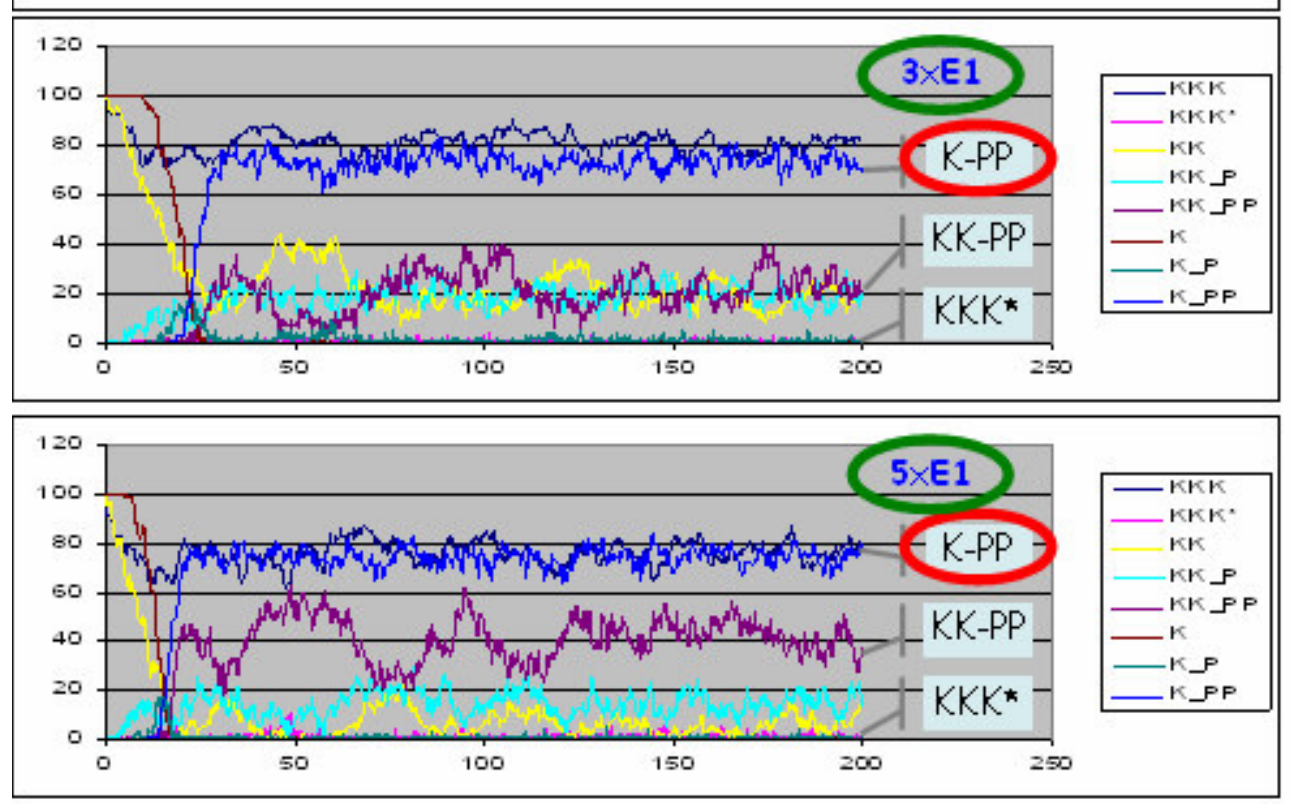


\section{MHC Class I Antigen Presentation}

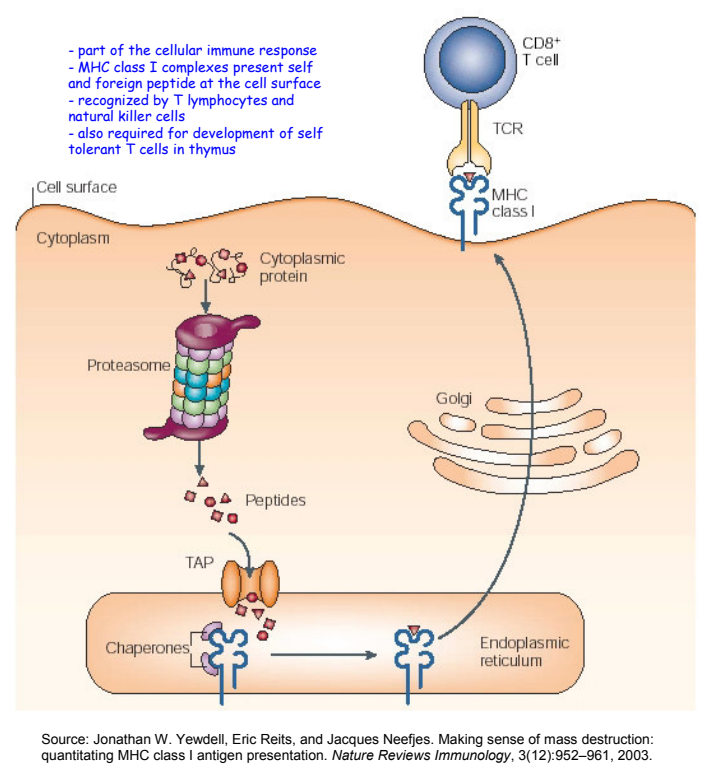

Model fit to three MHC class I heavy chain alleles

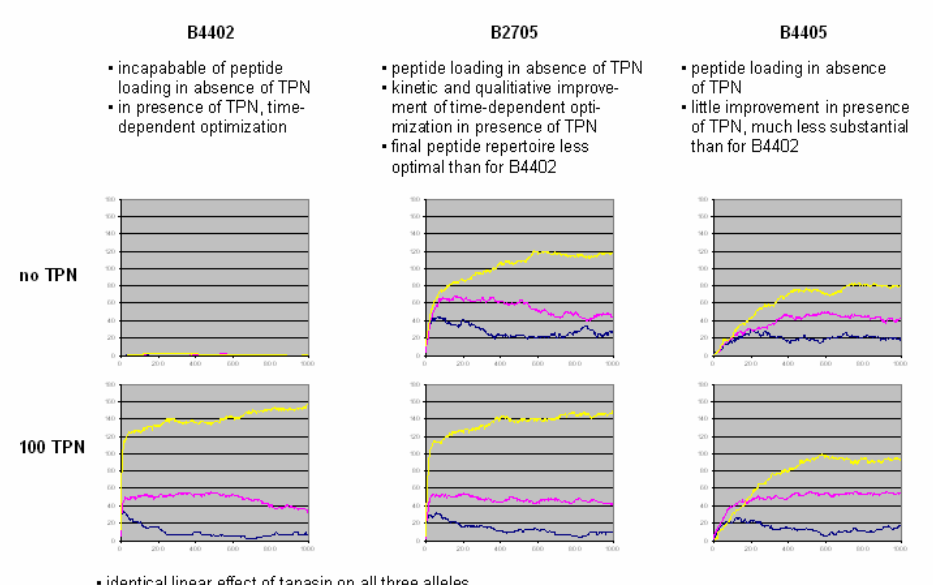

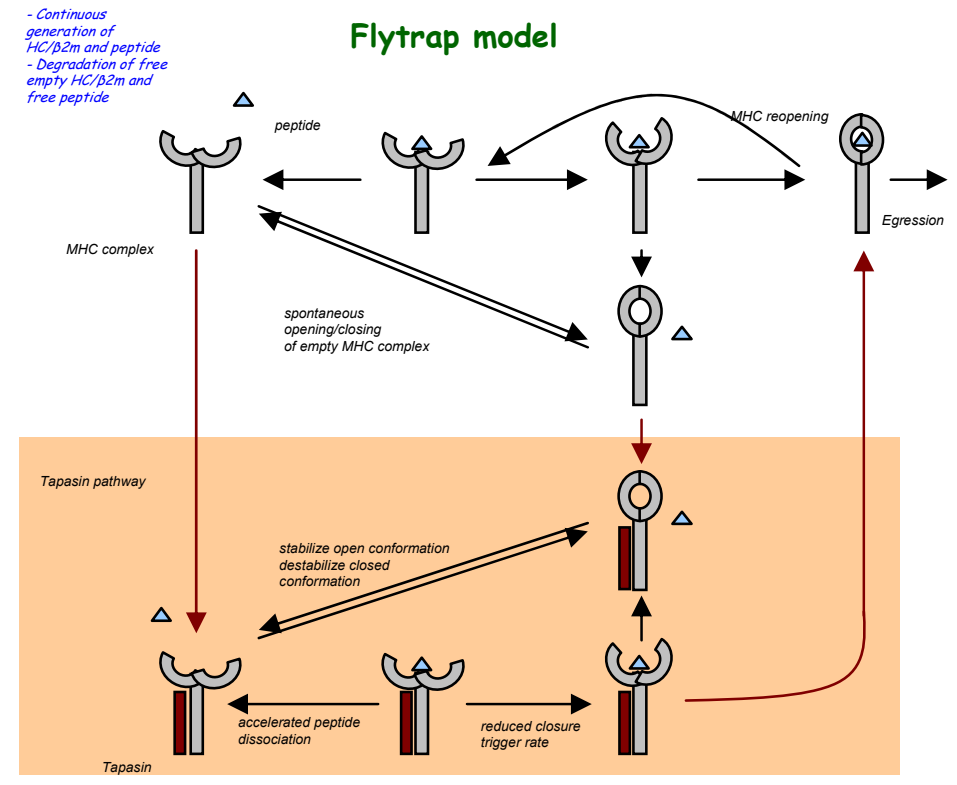

A stochastic pi-calculus model of MHC class I antigen presentation, Leonard Goldstein.

with Luca Cardelli and Andrew Phillips (Microsoft) and Tim Elliott and Joern Werner (U. Southampton) 


\section{The Gene Machine}

Pretty far from the atoms.

The "Central Dogma" of Molecular Biology
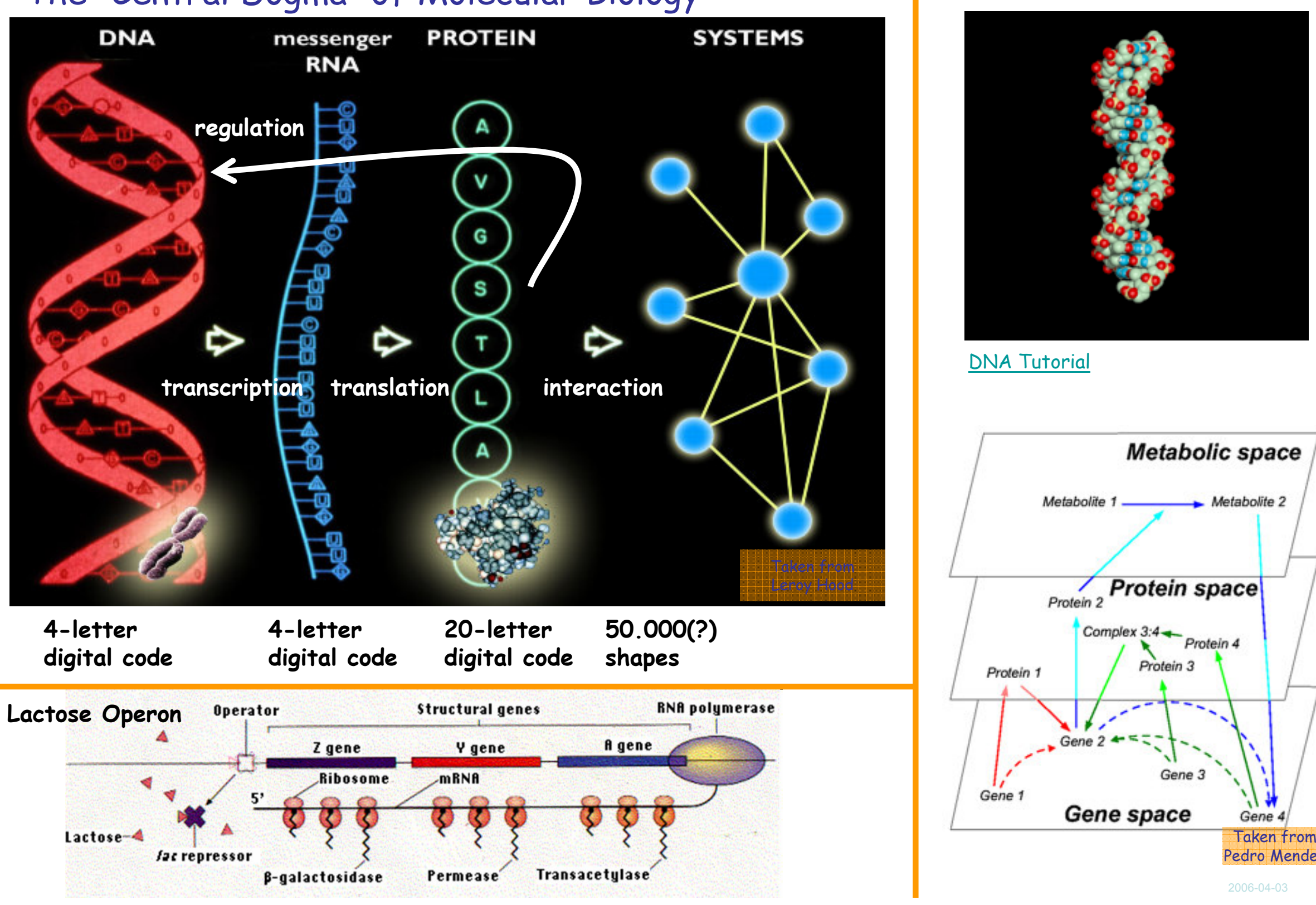

DNA Tutorial






\section{The Gene Machine "Instruction Set"}

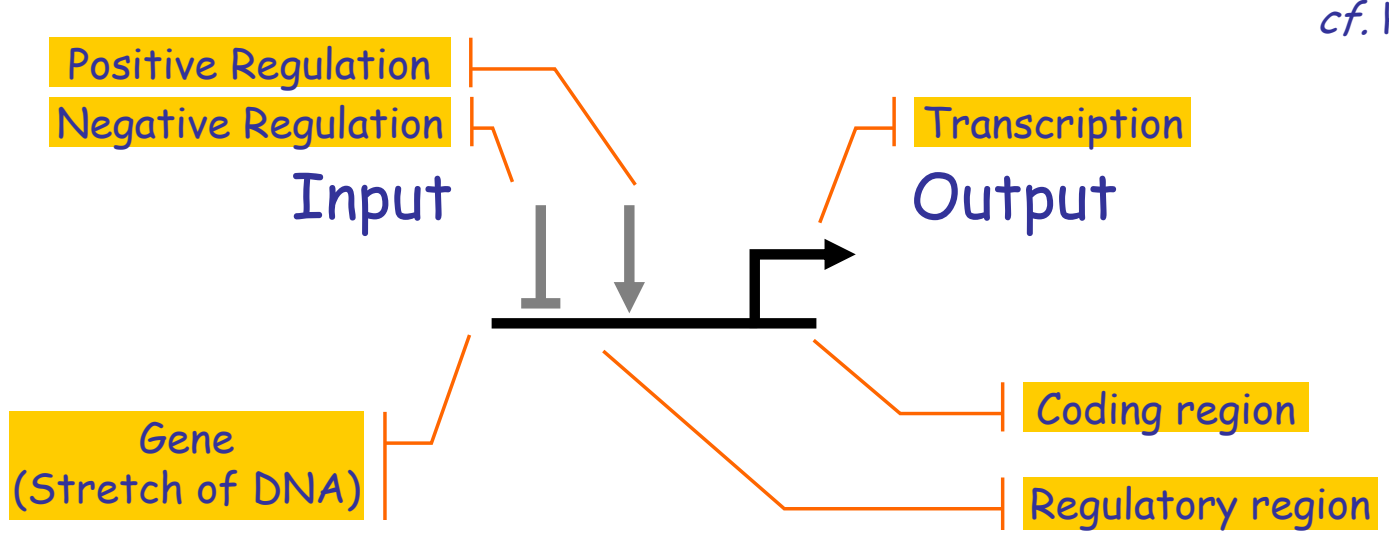

cf. Hybrid Petri Nets [Matsuno, Doi, Nagasaki, Miyano]

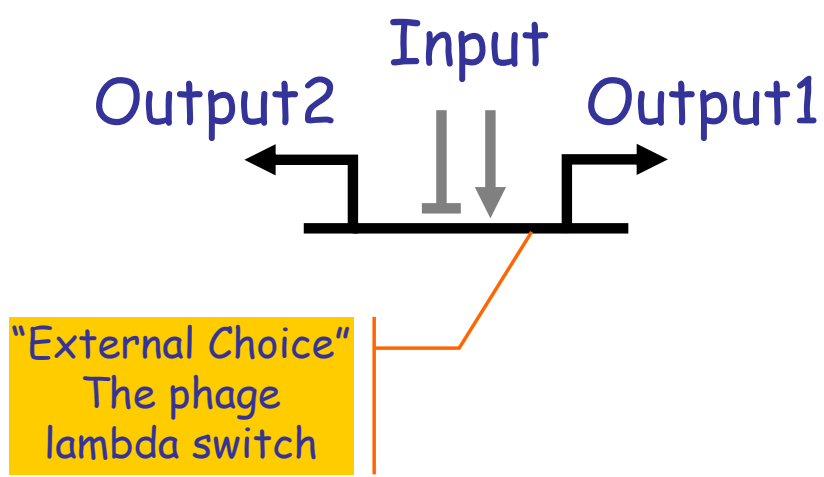

Regulation of a gene (positive and negative) influences transcription. The regulatory region has precise DNA sequences, but not meant for coding proteins: meant for binding regulators.

Transcription produces molecules (RNA or, through RNA, proteins) that bind to regulatory region of other genes (or that are endproducts).

Human (and mammalian) Genome Size

3Gbp (Giga base pairs) 750MB @ 4bp/Byte (CD)

Non-repetitive: $16 b p 250 M B$

In genes: $320 \mathrm{Mbp} 80 \mathrm{MB}$

Coding: $160 \mathrm{Mbp}$ 40MB

Protein-coding genes: $30,000-40,000$

M.Genitalium (smallest true organism) 580,073bp 145KB (eBook) E.Coli (bacteria): $4 M b p 1 M B$ (floppy) Yeast (eukarya): 12Mbp 3MB (MP3 song) Wheat 17Gbp 4.25GB (DVD) 


\section{Gene Composition}



Is a shorthand for:

Under the assumptions [Kim \& Tidor]

1) The solution is well-stirred

(no spatial dependence on concentrations or rates).

2) There is no regulation cross-talk.

3) Control of expression is at transcription level only (no RNA-RNA or RNA-protein effects)

4) Transcriptions and translation rates monotonically affect $m R N A$ and protein concentrations resp.

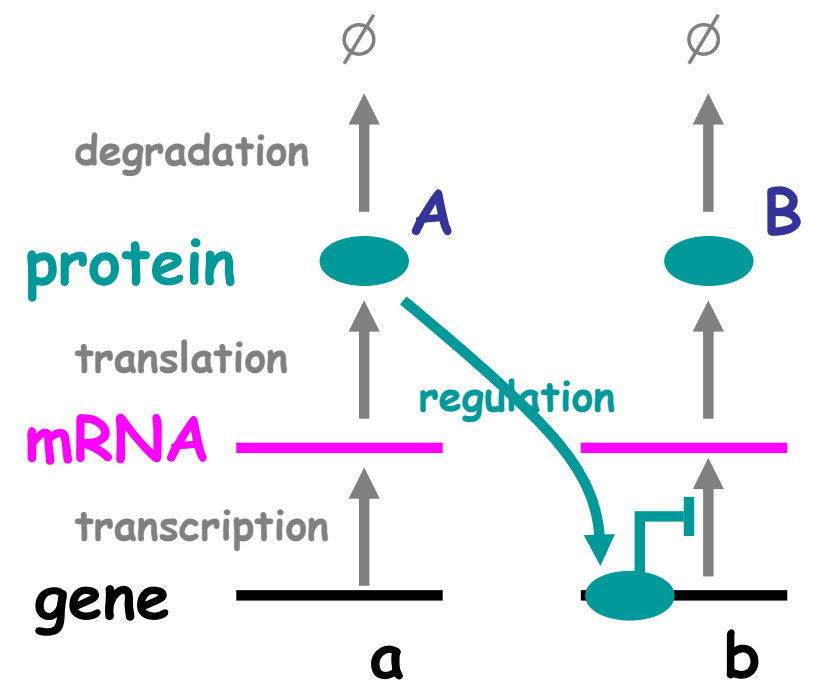

Ex: Bistable Switch


Ex: Oscillator
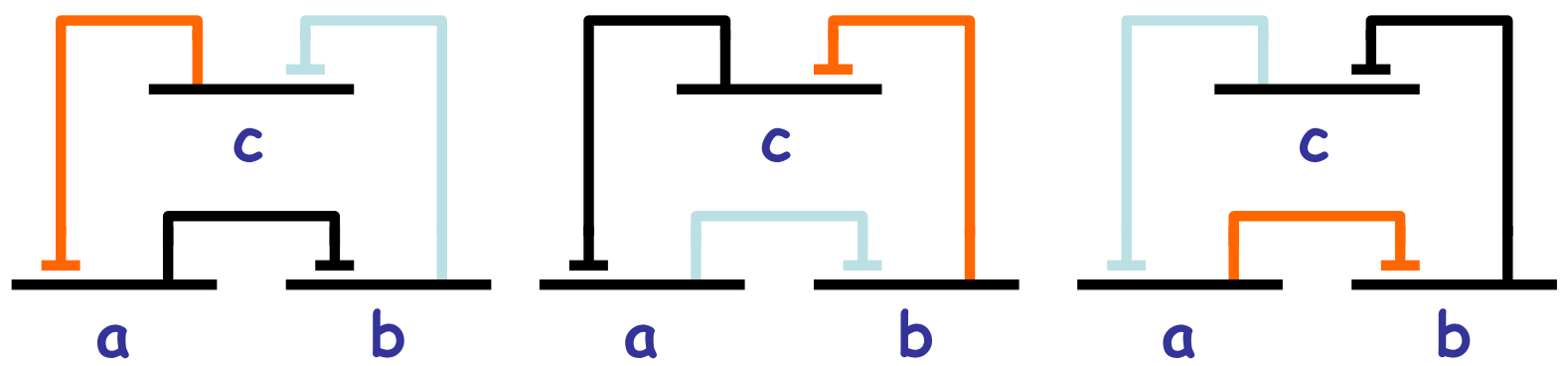

Expressed

Repressed

Expressing

b 


\section{Gene Regulatory Networks}

http://strc.herts.ac.uk/bio/maria/NetBuilder/
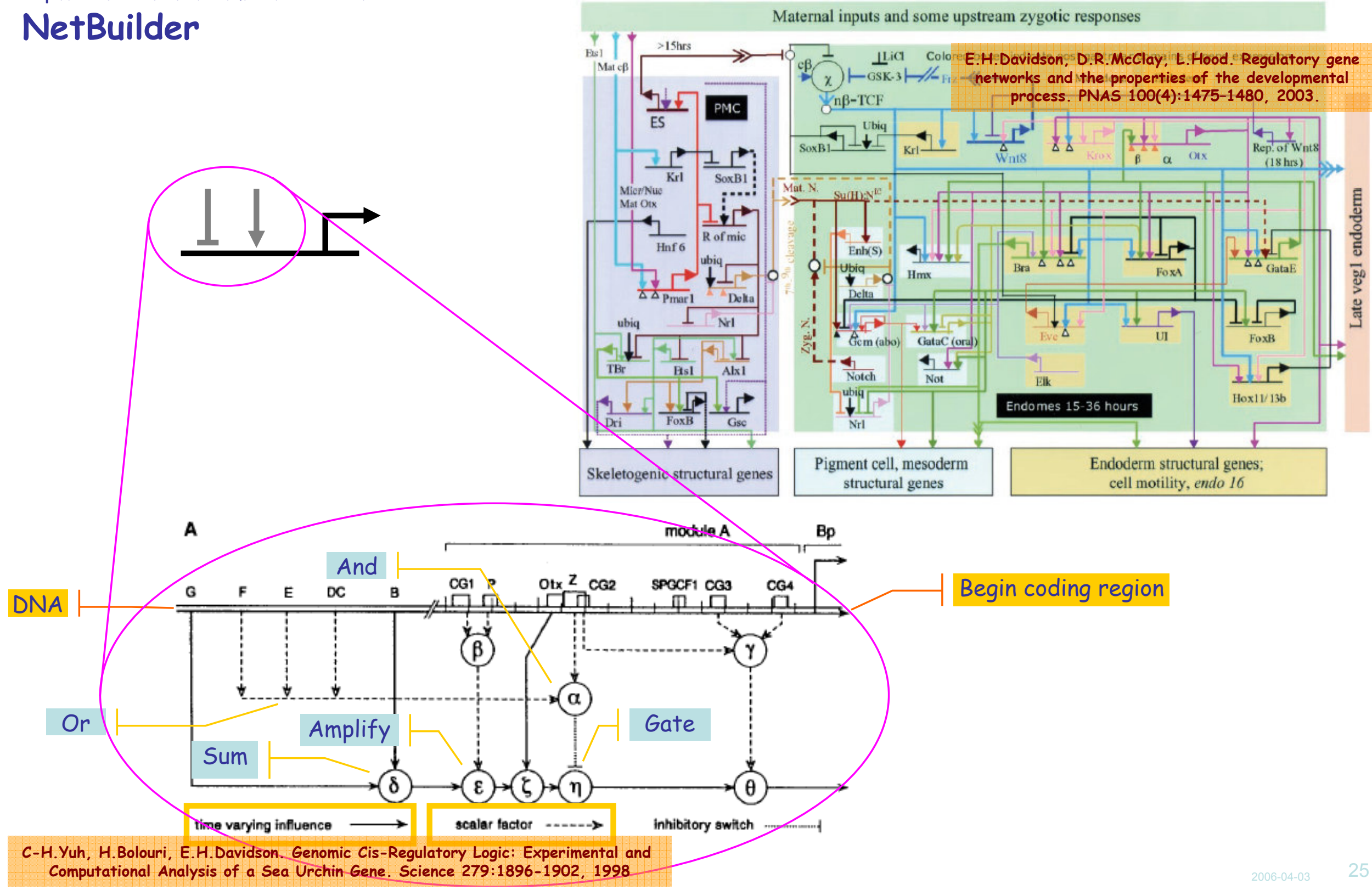


\section{The Programming Model}

- Strange facts about genetic networks:

- Not an operator algebra. The output of each gate is fixed and pre-determined; it is never a function of the input!

- Not term-rewriting, nor Petri nets. Inhibition is widespread.

- Not Communicating Sequential Processes. Feedback is widespread: asynchronous communication needed to avoid immediate self-deadlocks. Even the simplest gates cannot be modeled as a single synchronous automata.

- Not Message-Passing between genes. Messages themselves have behavior (e.g., they stochastically decay and combine), hence messages are processes as well.

- Not Data-Flow. Any attempt to use data-flow-style modeling seems doomed because of widespread loops that lead to deadlocks or unbounded queues. Data-flow tokens do not "decay" like proteins.

- How can it possibly work?

- Stochastic broadcasting. The apparently crude idea of broadcasting a whole bunch of asynchronous decaying messages to activate a future gate, means there are never any "pipeline full" deadlocks, even in presence of abundant feedback loops.

- Stochastic degradation. Degradation is fundamental for system stability, and at the same time can lead to sudden instability and detection of concentration levels. 


\section{Notations for the Gene Machine}

- Many of the same techniques as for the Protein Machine apply.

- Process Calculi, Petri Nets, TermRewriting Systems...

- But the "programming model" is different.

- Asynchronous stochastic control.

- Biologically poorly understood.

- Network "motifs" are being analyzed.
- Specific techniques:

- Hybrid Petri Nets

- [Matsuno, Doi, Nagasaki, Miyano] Gene Regulation

- Genomic Object Net www.genomicobject.net

- Gene Regulation Diagrams

- Mixed Gene-Protein Diagrams 


\section{Gene Gates and Circuits}

A gene gate
$a \underset{\text { neg }}{\longrightarrow} \mathrm{b}$

$$
\begin{aligned}
& \operatorname{neg}(a, b) \triangleq \\
& ? a_{r} ; \tau_{\eta} ; \operatorname{neg}(a, b)+ \\
& \tau_{\varepsilon^{\prime}}(\operatorname{tr}(b) \mid \operatorname{neg}(a, b)) \\
& \operatorname{tr}(p) \triangleq\left(! p_{r} ; \operatorname{tr}(p)\right)+\tau_{\delta}
\end{aligned}
$$

A genetic circuit (engineered in E.Coli)

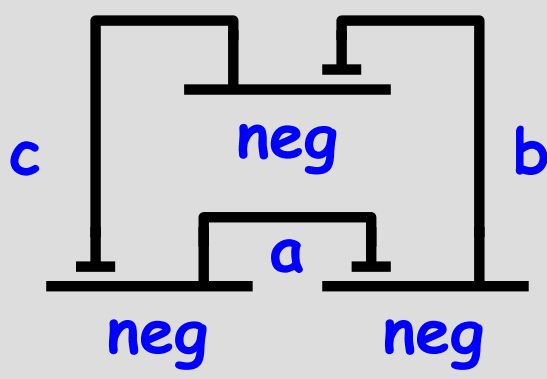

$$
\begin{aligned}
& \operatorname{neg}(a, b) \mid \\
& \operatorname{neg}(b, c) \mid \\
& \operatorname{neg}(c, a)
\end{aligned}
$$

\section{The stochastic- $\pi$ program}

$\begin{array}{ll}\text { val } \mathrm{dk}=0.001 & (\text { Decay rate } *) \\ \text { val inh }=0.001 & (\text { Inhibition rate } *) \\ \text { val cst }=0.1 & \text { (* Constitutive rate } *)\end{array}$

let $\operatorname{tr}(p: \operatorname{chan}())=$

$$
\text { do ! } p ; \operatorname{tr}(p) \text { or delayadk }
$$

let neg(a:chan ()$, b: \operatorname{chan}())=$

do ?a; delay@inh; neg $(a, b)$

or delay@cst; $(\operatorname{tr}(b) \mid \operatorname{neg}(a, b))$

(* The circuit *)

val bnd $=1.0 \quad$ (* Protein binding rate *)

new a@bnd:chan() new b@bnd:chan() new c@bnd:chan()

$\operatorname{run}(\operatorname{neg}(c, a)|\operatorname{neg}(a, b)| \operatorname{neg}(b, c))$

\section{A stochastic simulation (in SPiM)}

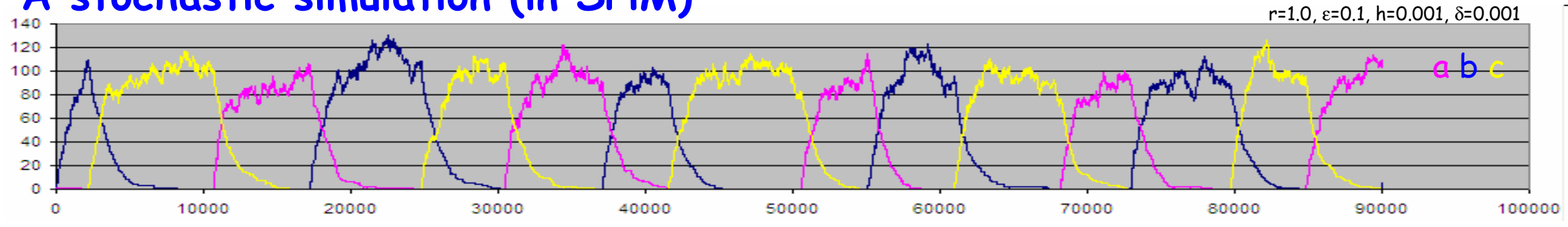




\section{Guet et al.: D038/lac-}

Combinatorial Synthesis of Genetic Networks, Guet, Elowitz, Hsing, Leibler, 1996, Science, May 2002, 1466-1470.
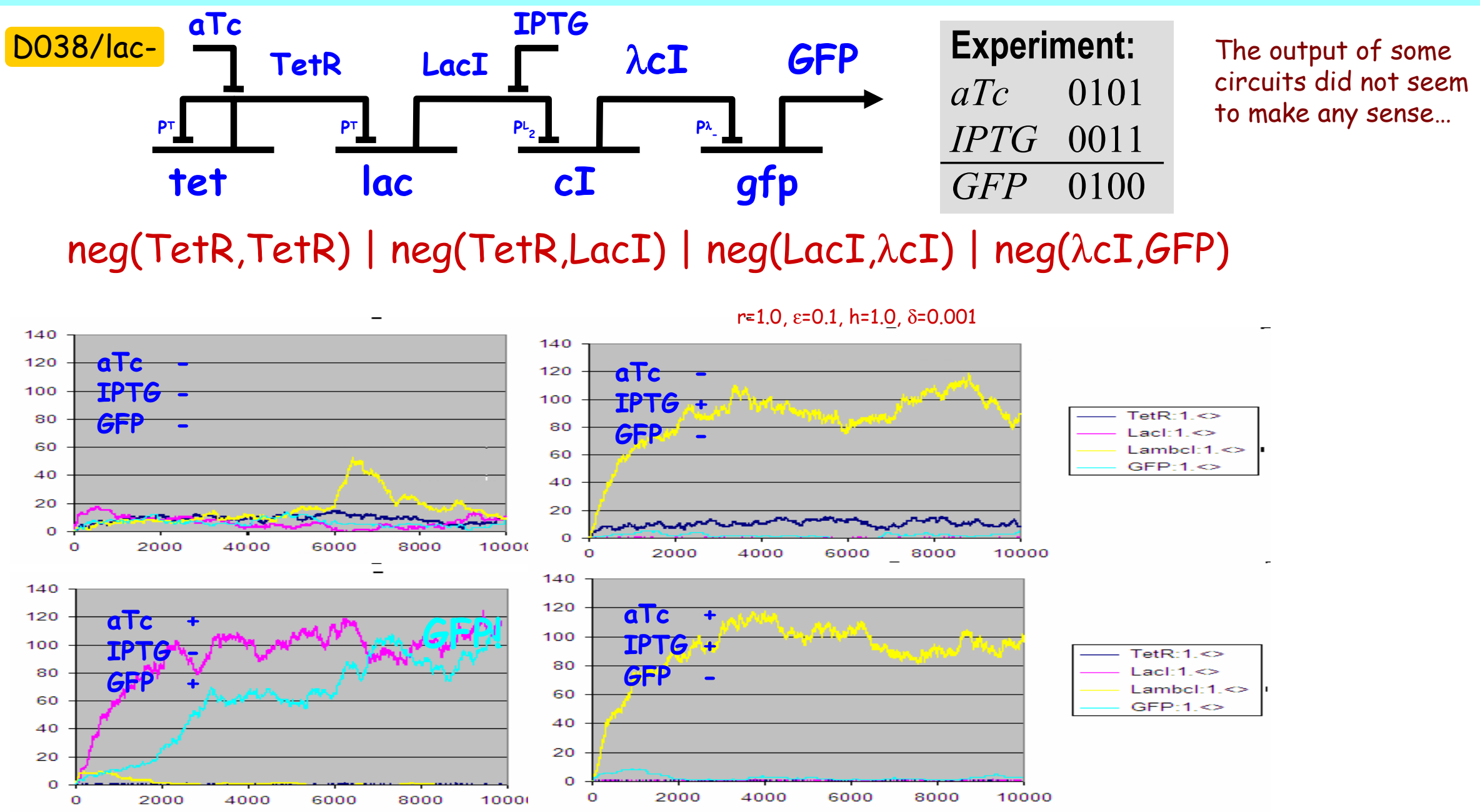

A Compositional Approach to the Stochastic Dynamics of Gene Networks, Ralf Blossey, Luca Cardelli, Andrew Phillips, TCSB, Springer, to appear. 


\section{The Membrane Machine Very far from 3. The Membrane Machine the atoms.}

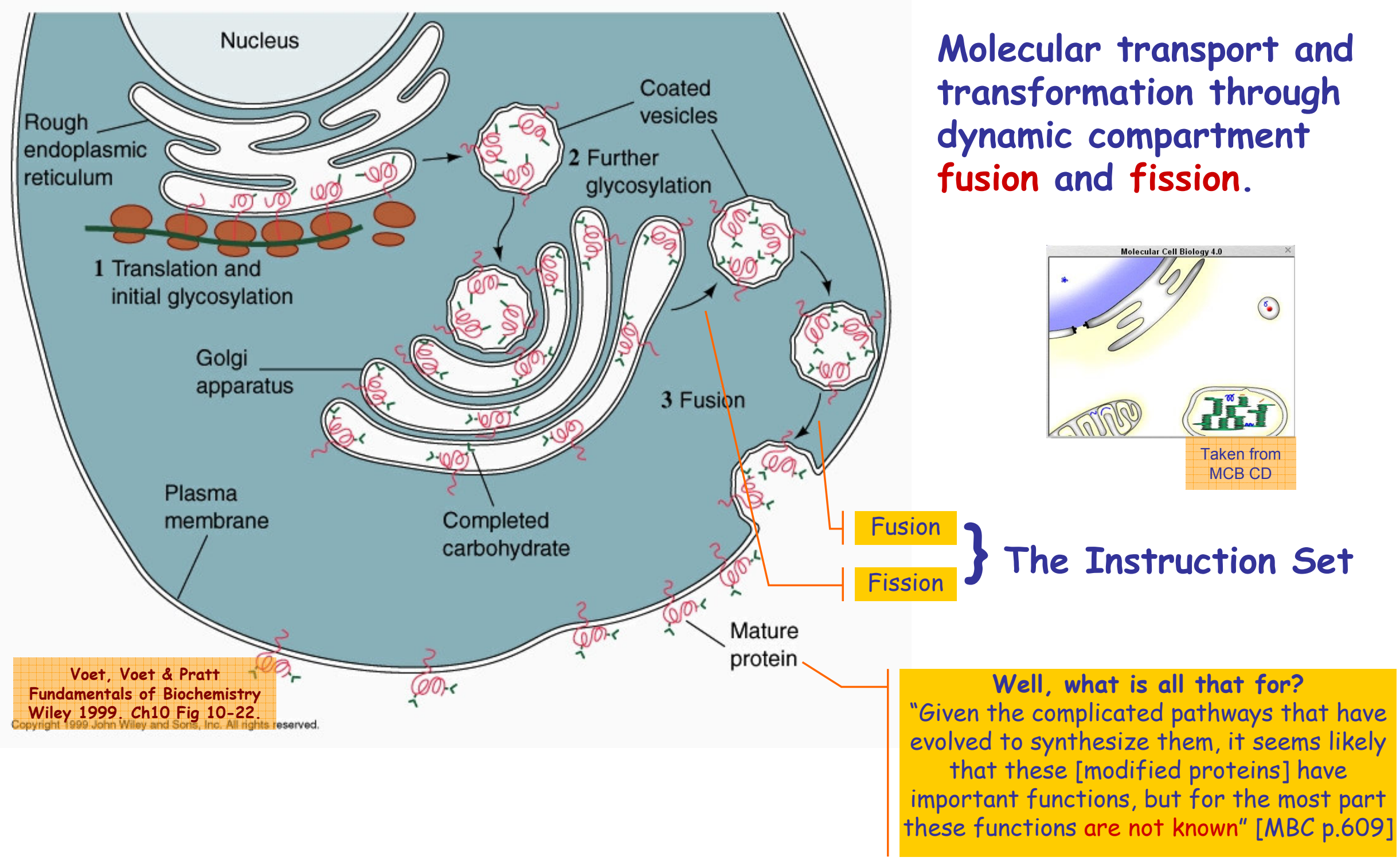




\section{Membrane Fusion}

Positive curvature to Negative curvature transition in 3D

Cell membrane

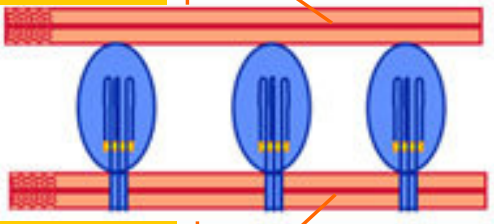

Virus membrane

1

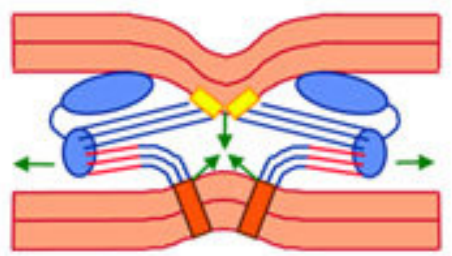

4



2



5

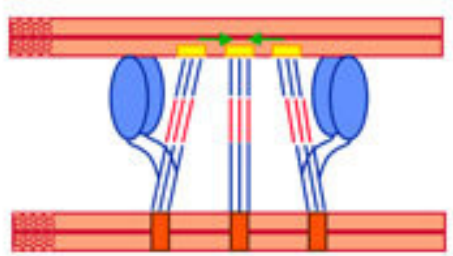

3



Aggressive fusion (virus)

By unknown mechanisms, the exoplasmic leaflets of the two membranes fuse" [MCB p745]

\section{Proposed sequence of events in $\mathrm{pH}$ sensitive hemagglutinin membrane fusion}



Cooperative fusion (vesicle)

"Fusion of the two membranes immediately follows prefusion, but precisely how this occurs is not known" [MCB p742] 


\section{Membrane Fission}

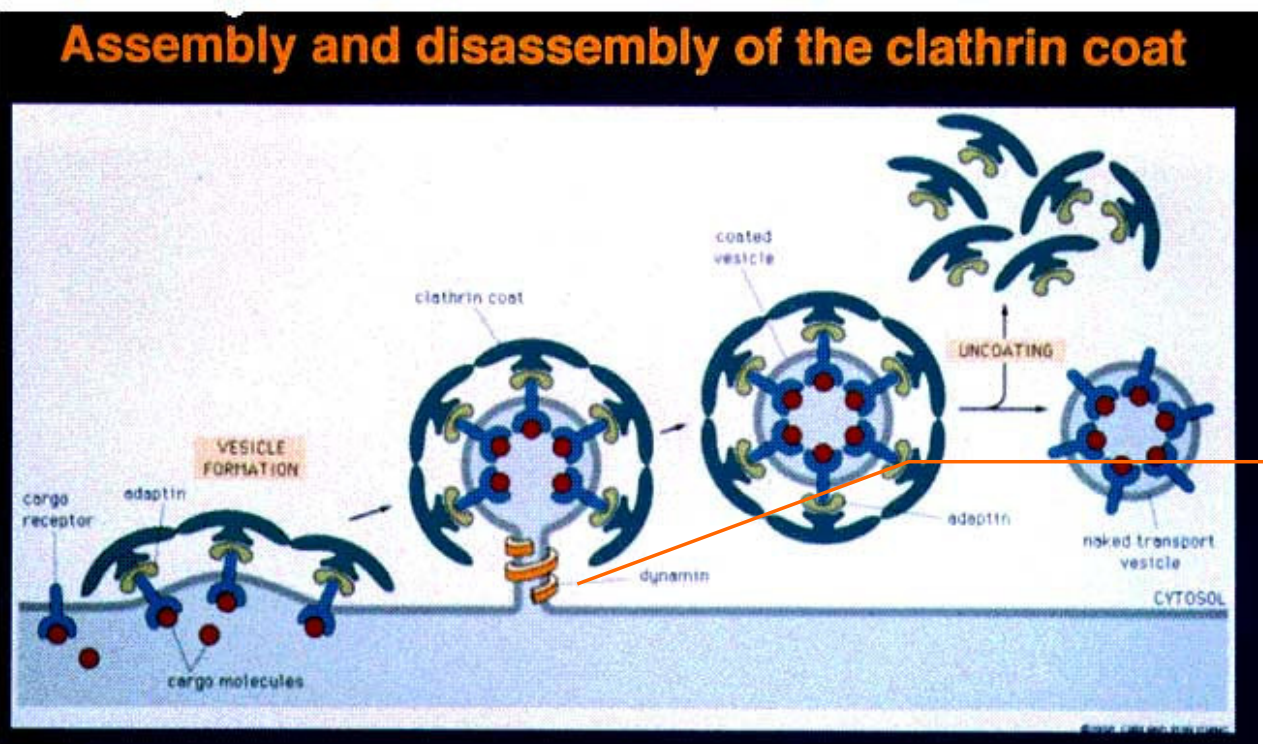

Vesicle

Formation

"Nonetheless, the actual

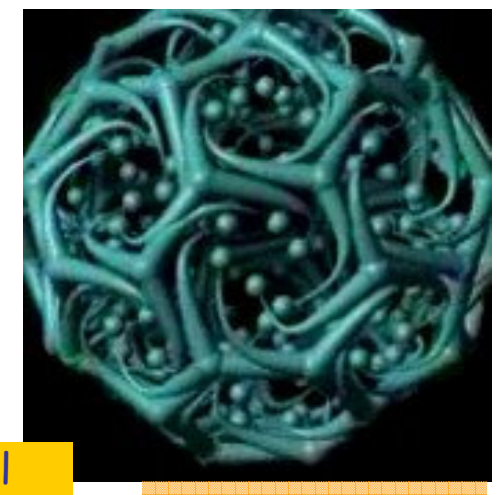
process whereby a segment of phospholipid bilayer is 'pinched off' to form a pit and eventually a new vesicle is still not understood" [MCB p.746]

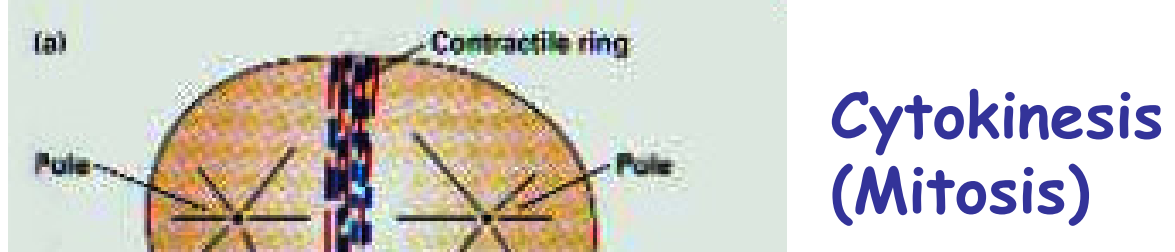




\section{The Membrane Machine "Instruction Set"}
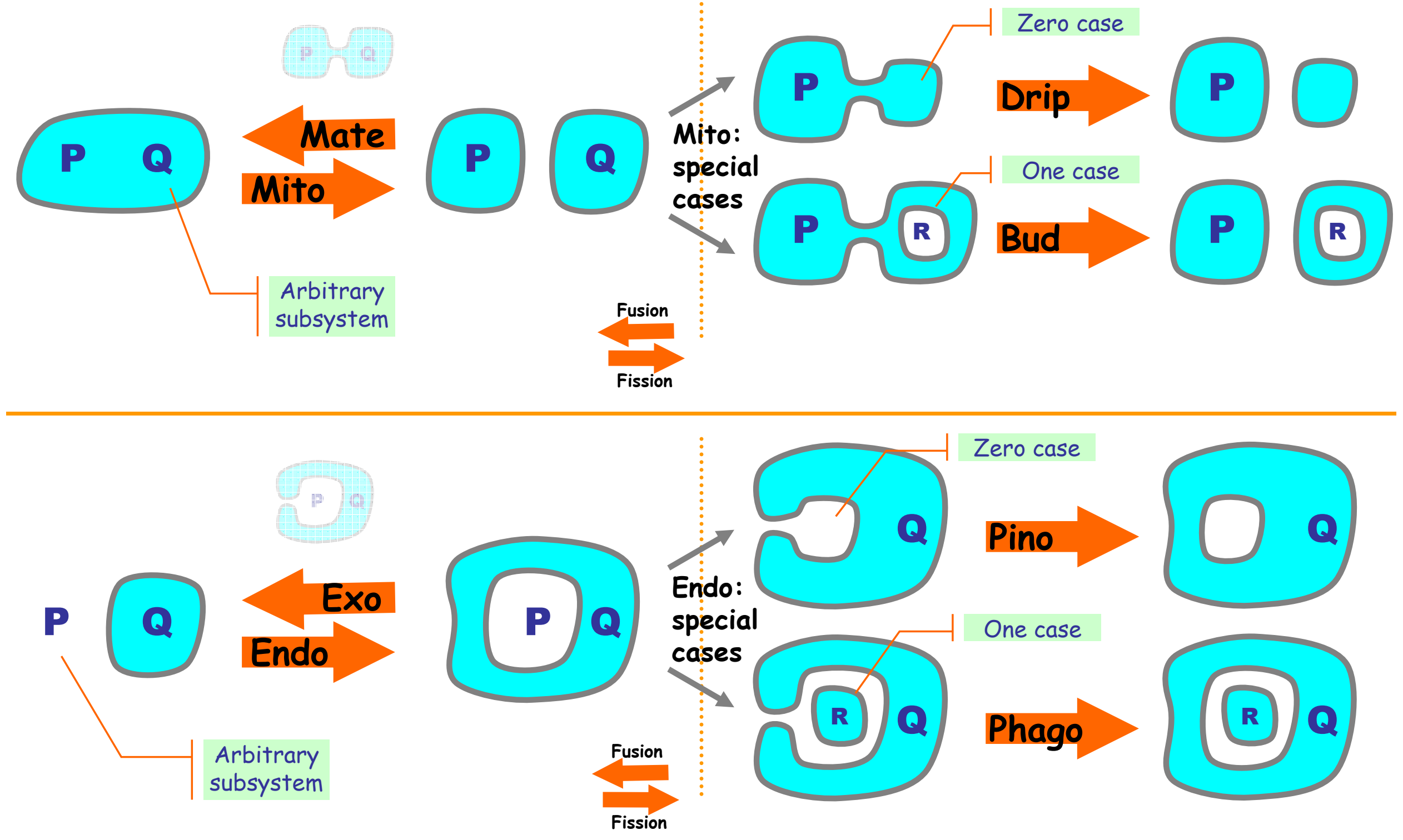


\section{$\ldots$ in $3 D$}

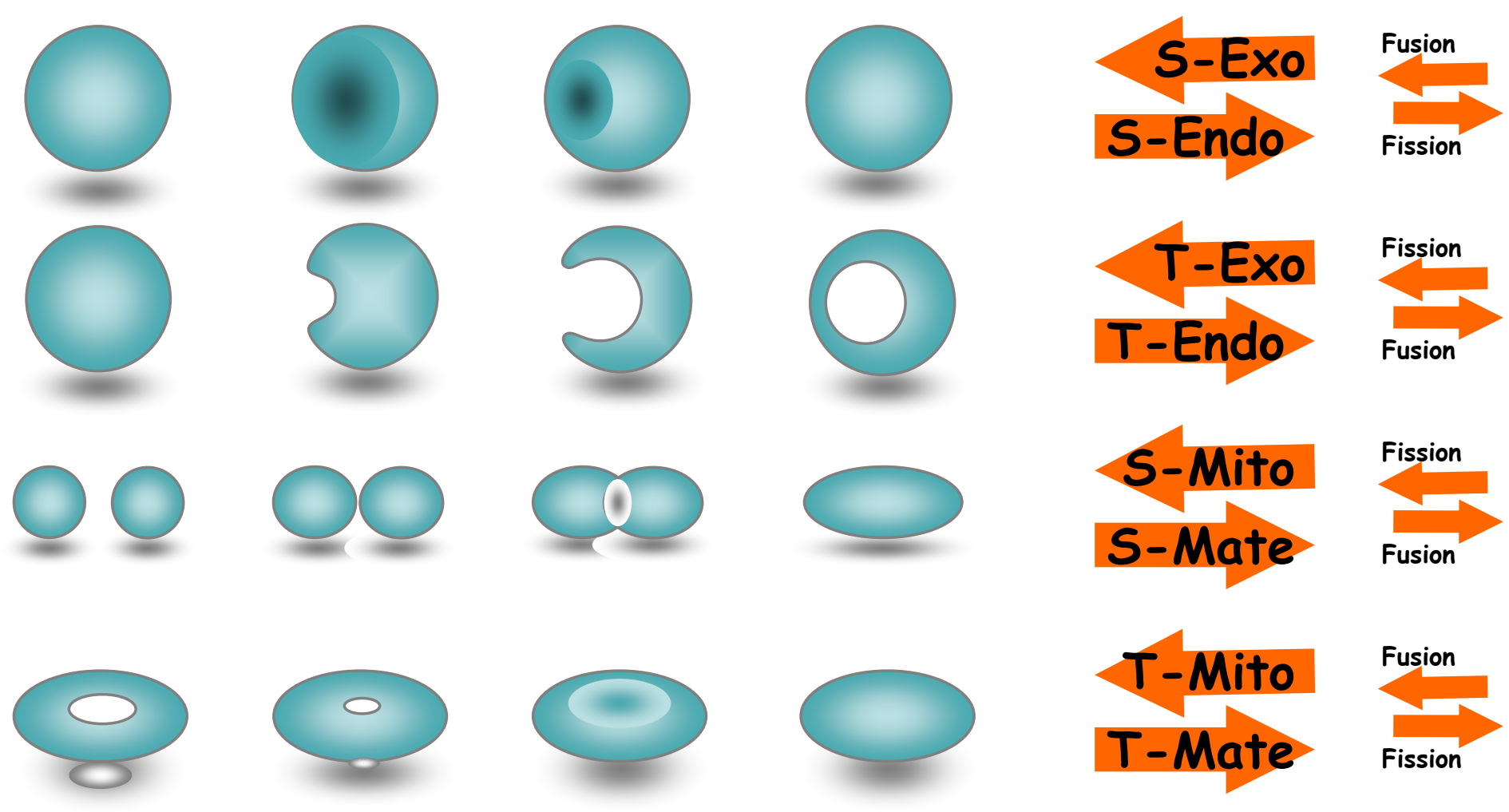




\section{Mito/Mate by 3 Endo/Exo}
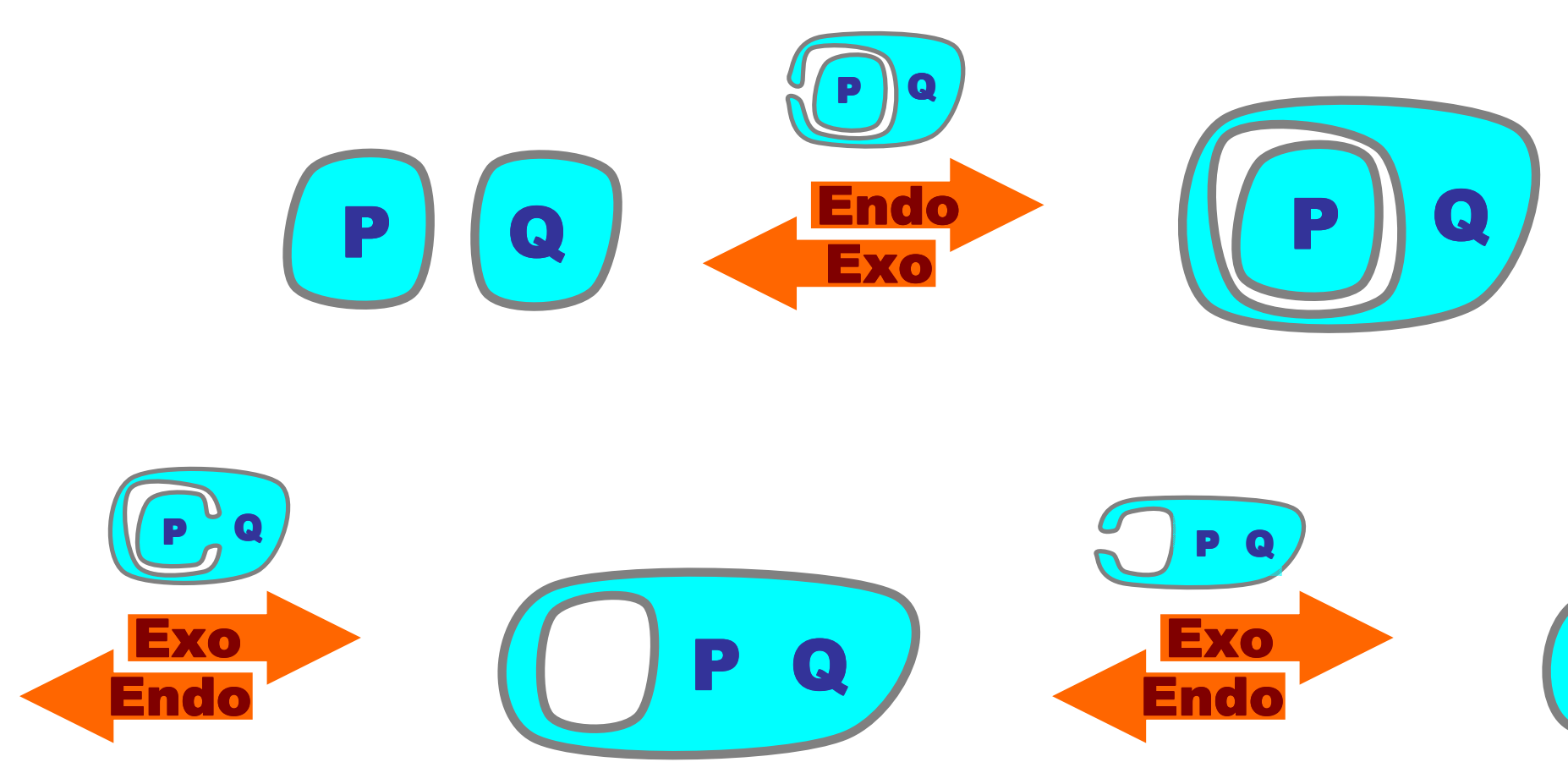


\section{Notations for the Membrane Machine}

- "Snapshot" diagrams

- In biology literature.

- P-Systems

- G.Paun uses ideas from the theory of grammars and formal languages to model "Membrane Computing" (book 2002).

http://psystems.disco.unimib.it/.
- BioAmbients

- An extension of BioSPI along Ambient Calculus lines (with more bio-relevant mobility primitives) to model dynamic compartments.

- Brane Calculi

- Computation on the membrane... 


\section{Membrane Algorithms}

Protein Production and Secretion

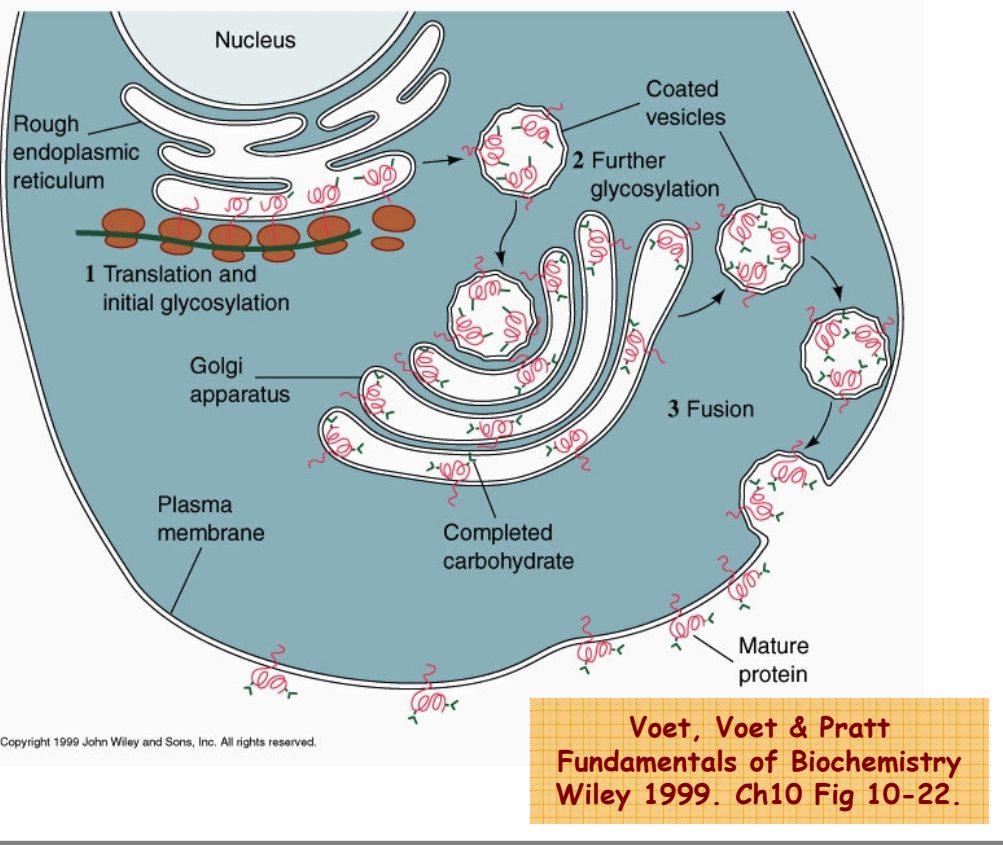

\section{Viral Replication}

Adapted from: B. Alberts et al. Molecular Biology of the Cell third edition p. 279.



LDL-Cholesterol Degradation






\section{Abstract Machines of Systems Biology}

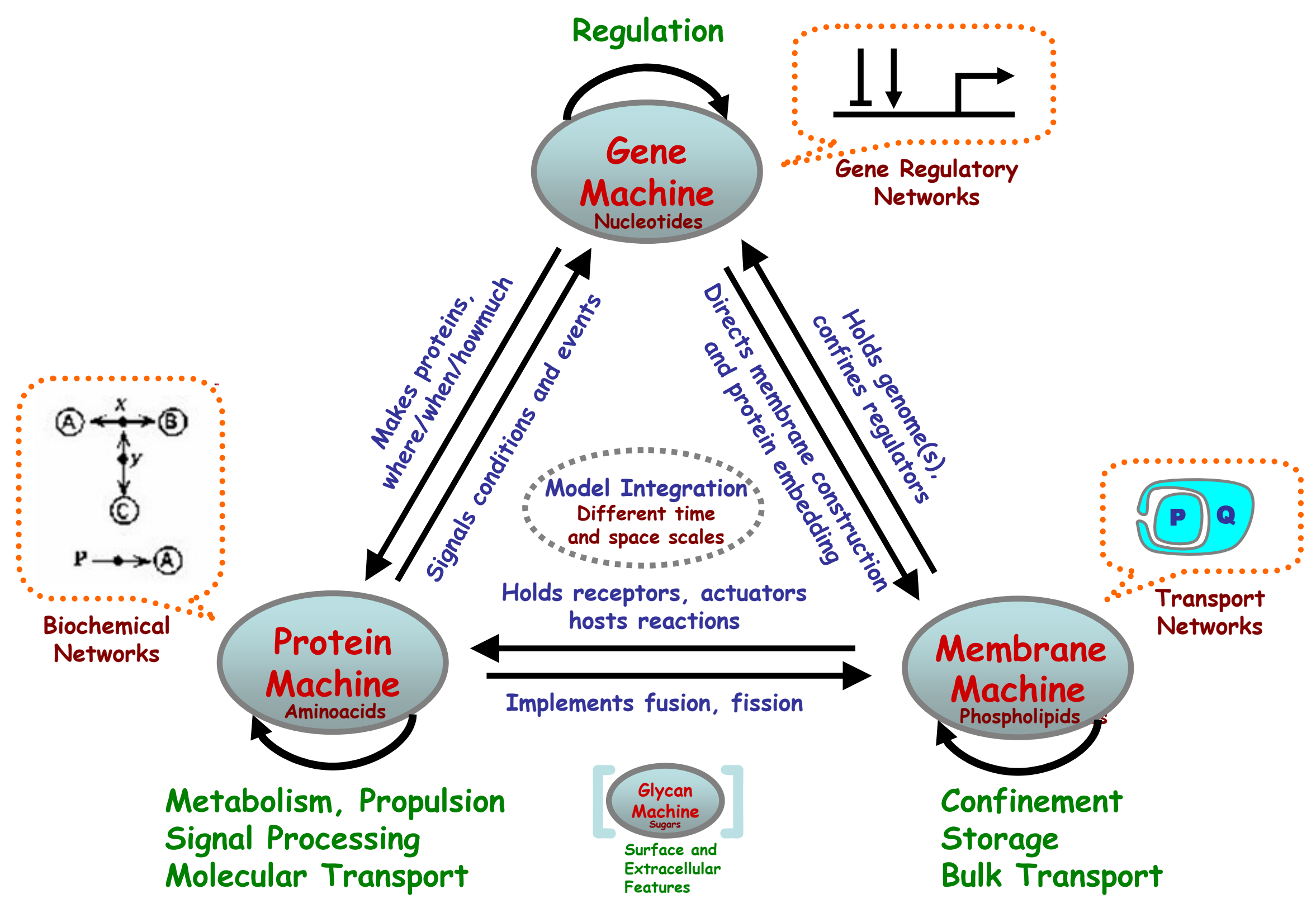




\section{Model Construction and Validation}




\section{Reactive Systems}

- Modeling biological systems

- Not as continuous systems (often highly nonlinear)

- But as discrete reactive systems; abstract machines where:

- States represent situations

- Event-driven transitions between states represent dynamics

- The adequacy of describing (discrete) complex systems as reactive systems has been argued convincingly [Harel]

- Many biological systems exhibit features of reactive systems:

- Discrete transitions between states

- Deep layering of abstractions ("steps" at multiple levels)

- Complexity from combinatorial interaction of simple components

- High degree of concurrency and nondeterminism

- "Emergent behavior" not obvious from part list 


\section{Model Validation: Simulation}

- Basic stochastic algorithm: Gillespie

- Exact (i.e. based on physics) stochastic simulation of chemical kinetics.

- Can compute concentrations and reaction times for biochemical networks.

- Stochastic Process Calculi

- BioSPi [Shapiro, Regev, Priami, et. al.]

- Stochastic process calculus based on Gillespie.

- BioAmbients [Regev, Panina, Silverma, Cardelli, Shapiro]

- Extension of BioSpi for membranes.

- Case study: Lymphocytes in Inflamed Blood Vessels [Leca, Priami, Quaglia]

- Original analysis of lymphocyte rolling in blood vessels of different diameters.

- Case study: Lambda Switch [Celine Kuttler, IRI Lille]

- Model of phage lambda genome (well-studied system).

- Case study: VICE [u. Pisa]

- Minimal prokaryote genome (180 genes) and metabolism of whole VIrtual CEII, in stochastic $\pi$-calculus, simulated under stable conditions for 40K transitions.

- Hybrid approaches

- Charon language [upenn]

- Hybrid systems: continuous differential equations + discrete/stochastic mode switching.

- Etc. 


\section{Model Validation: "Program" Analysis}

- Causality Analysis

- Biochemical pathways, ("concurrent traces" such as the one here), are found in biology publications, summarizing known facts.

- This one, however, was automatically generated from a program written in BioSpi by comparing traces of all possible interactions. [Curti, Priami, Degano, Baldari]

- One can play with the program to investigate various hypotheses about the pathways.

- Control Flow Analysis

- Flow analysis techniques applied to process calculi.

- Overapproximation of behavior used to answer questions about what "cannot happen".

- Analysis of positive feedback transcription regulation in BioAmbients [Flemming Nielson].

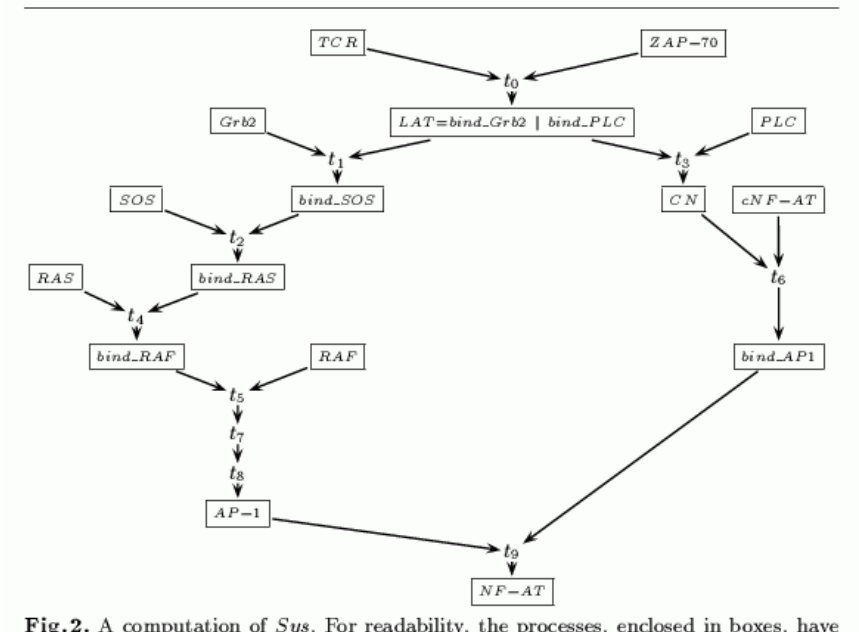

Fig.2. A computation of Sys. For readability, the processes, enclosed in boxes, have diagram resulting from the) arrows; their absence makes it explicit concurrent activities.

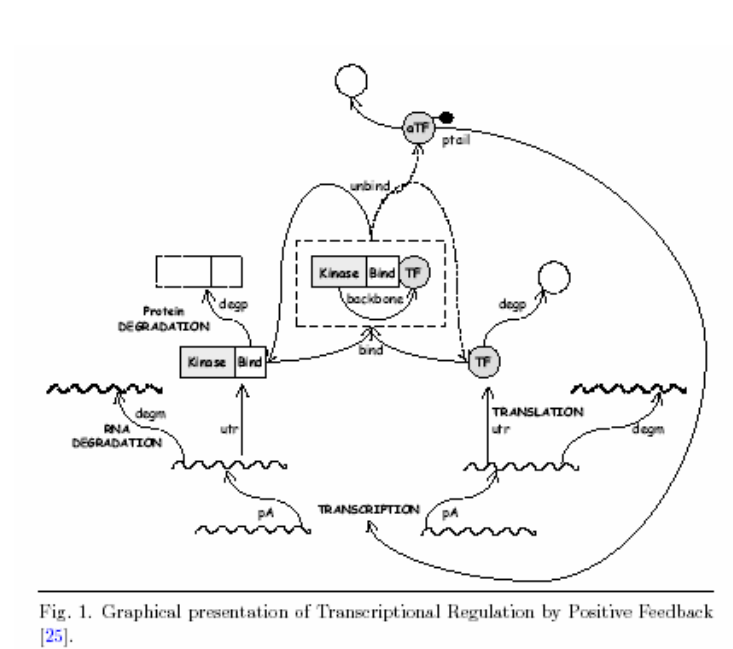

- Probabilistic Abstract Interpretation

- [DiPierro Wicklicky]. 


\section{Model Validation: Modelchecking}

- Temporal

- Software verification of biomolecular systems (NA pump)

- Analysis of mammalian cell cycle (after Kohn) in CTL. [Chabrier-Rivier Chiaverini Danos Fages Schachter]

- E.g. is state $S_{1}$ a necessary checkpoint for reaching state $S_{2}$ ?

- Quantitative: Simpathica/xssys

- Quantitative temporal logic queries of human Purine metabolism model.

Eventually(Always (PRPP $=1.7$ * PRPP1)

implies

steady_state()

and Eventually(Always(IMP $<2$ * IMP1))

and Eventually(Always(hx_pool $<10^{\star} h x \_$pool1)))



- Stochastic: Spring

- Designed for stochastic (computer) network analysis

- Discrete and Continuous Markov Processes.

- Process input language.

- Modelchecking of probabilistic queries. 


\section{What Reactive Systems Do For Us}

We can write things down precisely

- We can modularly describe high structural and combinatorial complexity ("do programming").

We can calculate and analyze

- Directly support simulation.

- Support analysis (e.g. control flow, causality, nondeterminism).

- Support state exploration (modelchecking).

We can visualize

- Automata-like presentations.

- Petri-Net-like presentations.

- State Charts, Live Sequence Charts [Harel]

- Hierarchical automata.

- Scenario composition.
We can reason

- Suitable equivalences on processes induce algebraic laws.

- We can relate different systems (e.g. equivalent behaviors).

- We can relate different abstraction levels.

- We can use equivalences for state minimization (symmetries).

\section{Disclaimers}

- Some of these technologies are basically ready (medium-scale stochastic simulation and analysis, medium-scale nondeterministic and stochastic modelchecking).

- Others need to scale up significantly to be really useful. This is (has been) the challenge for computer scientists.

Many approaches, same basic philosophy, tools being built:

$\Rightarrow$ Proc. Computational Methods in Systems Biology [2003-2005] 

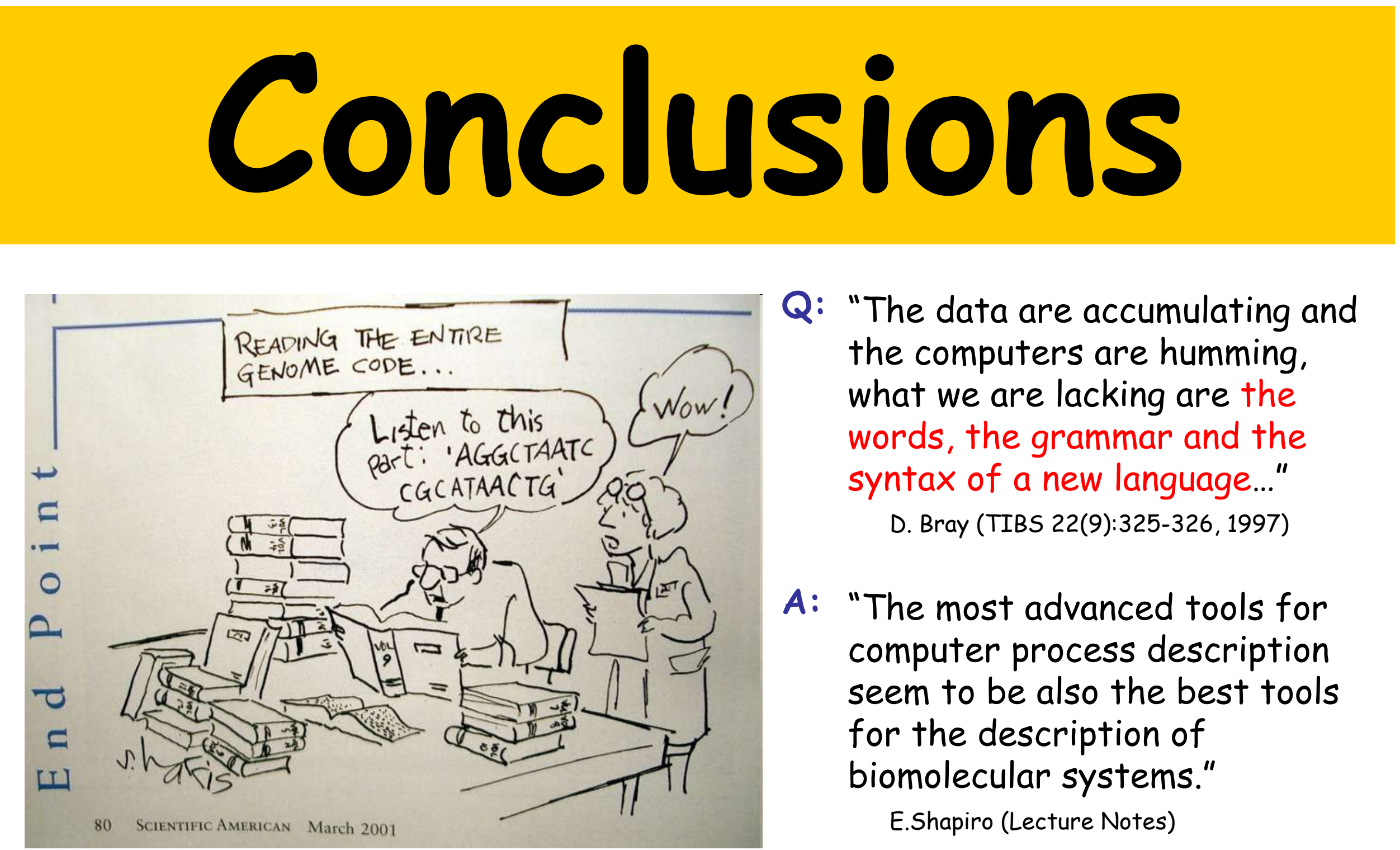

Q: "The data are accumulating and the computers are humming, what we are lacking are the words, the grammar and the syntax of a new language..."

D. Bray (TIBS 22(9):325-326, 1997)

A: "The most advanced tools for computer process description seem to be also the best tools for the description of biomolecular systems."

E.Shapiro (Lecture Notes) 


\section{References}

$[M C B]$ Molecular Cell Biology, Freeman.

$[M B C]$ Molecular Biology of the Cell, Garland.

[Ptashne] A Genetic Switch.

[Davidson] Genomic Regulatory Systems.

[Milner] Communicating and Mobile Systems: the Pi-Calculus.

[Regev] Computational Systems Biology: A Calculus for Biomolecular

Knowledge (Ph.D. Thesis).

Papers

BioAmbients

a stochastic calculus with compartments.

Brane Calculi

process calculi with computation "on" the membranes, not inside them. Bitonal Systems

membrane reactions and their connections to "local" patch reactions.

Abstract Machines of Systems Biology

the abstract machines implemented by biochemical toolkits.

www.luca.demon.co.uk/BioComputing.htm 


\section{Positions}

\section{Postdoc at Imperial College London: Centre for Integrative Systems Biology}

\section{Computational Modelling of Biological Processes}

Deadline for Applications: 10th February 2006

Applications are invited for the position of a research assistant/associate for up to three years to work on the application of process-modelling techniques to the signalling of phagocytosis. This position has been awarded to Dr Philippa Gardner and Dr Luca Cardelli (Microsoft Research Cambridge), funded by a large BBSRC/EPSRC grant to support a new Centre for Systems Biology at Imperial. It complements two equivalent positions (one for a biologist, one for a mathematician) in Centre for Molecular Microbiology and Infection \& Division of Cell and Molecular Biology, to investigate the spatio-temporal control of phagocytic signalling during uptake of bacteria. We expect the three researchers to work closely together.

Applicants should complete an application form, downloadable from

http://www.imperial.ac.uk/employment/academicform.htm. Applications will not be accepted unless they are on the correct form and clearly marked with the Job Reference Number PG Bio 05. The application form should be accompanied by a full CV with names and addresses of 3 referee and should be sent to: Mrs Nicola Rogers Department of Computing Imperial College London South Kensington Campus London, SW7 2 AZ UR Email: n.c.rogers@imperial.ac.uk.

\section{Various positions in Trento:}



http://www.msr-unitn.unitn.it



